\title{
The Safety of Medicinal Plants Used in the Treatment of Vitiligo and Hypermelanosis: A Systematic Review of Use and Reports of Harm
}

This article was published in the following Dove Press journal: Clinical, Cosmetic and Investigational Dermatology

\section{Irshad Hussain}

Institute of Pharmacy, Shaheed Mohtarma Benazir Bhutto (SMBB) Medical

University, Larkana, Pakistan
Correspondence: Irshad Hussain Institute of Pharmacy, Shaheed Mohtarma Benazir Bhutto (SMBB) Medical University, 7 Doctors Colony, Along VIP Road, Larkana, 77I50, Sindh, Pakistan Tel +92 3। 272 I4I I2

Email irshadpharmacist@yahoo.com
Introduction: Vitiligo is disfiguring and devastating condition that can humans feel stigmatic and devalued. Melasma is a general condition of hyperpigmentation particularly involving the face. The pigmentation disorders of vitiligo (hypopigmentation or depigmentation) and melasma (Hypermelanosis) are common among the world's population (around $1 \%$ for vitiligo).

Objective: The identification of medicinal plants used in the treatment of vitiligo and hypermelanosis. A systematic literature review on harms associated with the medicinal plants used in the treatment of vitiligo and hypermelanosis. To review and summarize information on reported adverse drug reactions (ADRs) associated with these medicinal plants contained in (where access is available) national and global individual case safety report databases.

Methods: A systematic review of the literature with special reference to all types of clinical trial and case reports using biomedical databases including Medline, EMBASE, Scopus, International Pharmaceutical Abstracts and so forth to identify medicinal plants alone or as an adjuvant with other treatments and their safety/tolerability in the treatment of vitiligo and Hypermelanosis. Other sources of this search were medicinal plants text books, pharmacopoeias and authentic websites discussing possible treatments for vitiligo/hypermelanosis. It also included databases such as VigiAccess containing data from spontaneous reporting schemes for ADRs.

Results: A total of 55 articles (47 clinical trials and 8 case reports) met the inclusion criteria. Some trials did not reported safety information, some did report, but not very well. Reports of blistering, erythema, acute hepatitis and mutagenesis with Psoralea corylifolia. Adverse effects of erythema (mild to severe), phototoxic reactions, mild raise in liver transaminases, gastrointestinal disturbances, burns, itching, scaling, depigmented macules, pruritis, and giddiness with the use of psoralens. Khellin-related erythema, perilesional hyperpigmentation, gastrointestinal disturbances, mild raise in liver transaminases and orthostatic complaints. Infrequent side effects with Ginkgo biloba. Lower grade of erythema and edema reported with the use of Polypodium leucotomos.

Conclusion: Primarily the retrieved clinical studies were efficacy oriented and safety parameters were secondary in priority whilst the general protocol of clinical trials requires the screening of drugs/medicinal plants on the basis of safety studies before testing the clinical aspects of efficacy. Thereby it is recommended that efficacy studies may be followed once the safety has been established for a particular medicinal plant in treating vitiligo and hypermelanosis.

Keywords: vitiligo, hypermelanosis, skin diseases, safety of medicinal plants, harm of herbs 


\section{Introduction}

Vitiligo is disfiguring and devastating condition that can make humans feel stigmatic and devalued. ${ }^{1}$ Melasma is a general condition of hyperpigmentation and particularly involves the face. ${ }^{2}$ The pigmentation disorders of vitiligo (hypopigmentation or de-pigmentation) and melasma (Hypermelanosis) are common among the world's population (around 1\% for vitiligo). The exact cause of both of the diseases is unclear, though various factors have been suggested in predisposition of vitiligo and melasma. Both of the conditions negatively effect the physical image of the affected individual, and there have been major cosmetic concerns, particularly for females. This affects them psychologically and economically by increasing their visits to dermatology/skin care clinics. ${ }^{1,2}$ The use of natural products (complimentary/alternative medicines) for chronic health conditions is common and individuals may seek this approach for the treatment of vitiligo and melasma. Natural health products are promoted for use in these conditions. For the past few decades these products have been gaining popularity and there is an increase in demand because of their vast chemical diversity. These are believed to be relatively safe, reliable, easily accessible, and affordable to the public. ${ }^{3}$ There have been studies regarding the use of medicinal plants in the conditions of vitiligo and hypermelanosis, but these were found to have limited evidence to support their efficacy in these uses. ${ }^{4,5}$ Generally, public perception, particularly in underdeveloping countries, that topical/oral use of herbs has no untoward effects and thus there is lack of proper rules and regulations regarding the monitoring of manufacturing and sales of these natural pharmaceutical products. Plenty of beauty creams/ointments are advertized in print/electronic media and are available over the counter and many herbal practitioners use for them. ${ }^{6}$ There are fewer clinical investigations of medicinal plants with respect to their safety when used in the vitiligo and hypermelanosis. To the best of the author's knowledge there has been no systematic research previously published. Thus, the novel systematic review has focused on exploring reports relating to possible harm associated with medicinal plants and some of the constituents derived from plants (psoralens) used in the treatment of vitiligo and hypermelanosis. The aims of the project were to identify and summarize harm associated with the medicinal plants used in the treatment of vitiligo and hypermelanosis through literature searches from databases.

\section{Methods}

\section{Time Frame}

Six months.

\section{Setting}

School of Pharmacy, University of Auckland, New Zealand.

\section{Procedure}

1. The following procedure was adopted to identify medicinal plants used in the treatment of vitiligo and hypermelanosis and their mode of use (topical or oral) for this treatment.

1a. The first step was to conduct a literature search to identify medicinal plants used in vitiligo/melasma (V/M). A systematic review of the literature was carried out using biomedical databases, including Medline, EMBASE, Scopus, International Pharmaceutical Abstracts, and so on to identify medicinal plants used in the treatment of vitiligo and hypermelanosis. Once the results were obtained (August 7, 2017), a search of the titles and abstracts to find the papers which discussed medicinal plants in V/M. The title and the abstract of the particular research paper was viewed to extract the key words representing the botanical or common name of that plant used to treat $\mathrm{V} / \mathrm{M}$. The plants included were either those having reports of clinical usage or those having in vitro anti-vitiligo/anti-melasma activity. After doing this, there was compilation of a table (medicinal plants used in vitiligo and/or melasma) listing the names of the medicinal plants used in $\mathrm{V} / \mathrm{M}$, and some other details (e.g., other names for the plants, use in $\mathrm{V} / \mathrm{M}$, the reference, method of preparation/delivery, i.e., topical or internal.

1b. Some other sources were also searched (e.g., authoritative medicinal plant textbooks, publicly available websites and pharmacopoeia) where possible to see if other medicinal plants were listed as being used in V/M and added them to the Table.

Books to be searched were those available at MedicinesComplete especially Herbal Medicine, Stockley's Herbal Medicines Interactions, Clarke's Analysis of Drugs and Poisons, Martindale and Melasma and vitiligo in brown skin. Pharmacopoeias to be searched out were Indian pharmacopoeia (IPC) and Hamdard pharmacograph. 
Table I Publicly Available Websites

\begin{tabular}{|c|c|c|}
\hline $\begin{array}{l}\text { Serial } \\
\text { No. }\end{array}$ & Publicly Available Website (URL) & Name of Organization \\
\hline 1 & https://nccih.nih.gov/health/herbsataglance.htm, & National center for complimentary and integrative health $(\mathrm{NIH})$ \\
\hline 2 & https://www.nps.gov/plants/medicinal/ & Medicinal Plant Working Group (MPWG) \\
\hline 3 & http://medicinalplantgenomics.msu.edu/ & Medicinal Plants Genomics resource. \\
\hline 4 & http://www.pfaf.org/user/default.aspx & Plants for a future (PFAF) \\
\hline 5 & http://abc.herbalgram.org/site/PageServer & American botanical council (ABC) \\
\hline 6 & https://www.medicinalplants-pharmacognosy.com/ & $\begin{array}{l}\text { Pharmacognosy, s Topics } \\
\text { Medicinal Plants. }\end{array}$ \\
\hline 7 & http://www.medicinalplants.in/ & The national medicinal plants board (NMPB) \\
\hline 8 & $\begin{array}{l}\text { http://www.healthy.net/clinic/therapy/herbal/herbic/herbs/index. } \\
\text { asp }\end{array}$ & Healthy.net \\
\hline 9 & http://www.webmd.com/ & WebMD \\
\hline
\end{tabular}

\section{Publicly Available Websites}

There had been a general look on publicly available websites and the following sites (see Table 1) have been found leading, informative and relevant to retrieve the information on safety and harm of medicinal plants.

1. The following procedure was performed to undertake a literature review on harm associated with the use of medicinal plants in the treatment of vitiligo and hypermelanosis. 2a. Once part $1 \mathrm{a} / 1 \mathrm{~b}$ was finished, the list of medicinal plants was used in the table to do another literature search. This involved entering all the plant names (as well as their common names) and combine with "OR,. To findclinical information on harm, the search was limited to all the clinical information types (i.e., all the clinical trial, case reports, systematic reviews, etc.). The results of this search were combined using "AND, with the list obtained by combining the disease terms with "OR,, to get the relevant data. Other limits were applied at this stage, e.g., "human,, and in the English language. Then the pooled medicinal plants group were combined with the "clinical" group with "AND,., This then provided the set of literature to work on for Aim 2, i.e., the systematic review on harms associated with the use of med plants in V/M. Psoralens were included in the review by adding the search terms for these into plant names at this stage (Figure 1).

2b. To look through the titles and abstracts of this group and to select the papers that contained information on harms/adverse effects came from clinical trial reports, or from case reports with these medicinal plants, or other types of papers. Using the relevant papers to summarise the information on harms/adverse effects for each of the medicinal plant.

1. Using the list of medicinal plants in the Table from $1 \mathrm{~b}$ above, there was a search of the ADR databases to find reports of suspected adverse drug reactions (ADRs) submitted to national medicines agencies/pharmacovigilance centres. Systematic searches of WHO VigiAccess database (the publicly available version of the WHO's individual case safety report database) and other databases containing data from spontaneous reporting schemes for ADRs, such as EudraVigilance (the European database of suspected ADR reports), SMARS (Suspected Medicine Adverse Reaction Scheme (NZ).

\section{Inclusion Criteria}

Articles containing information on adverse events or other safety-related information associated with the use of 


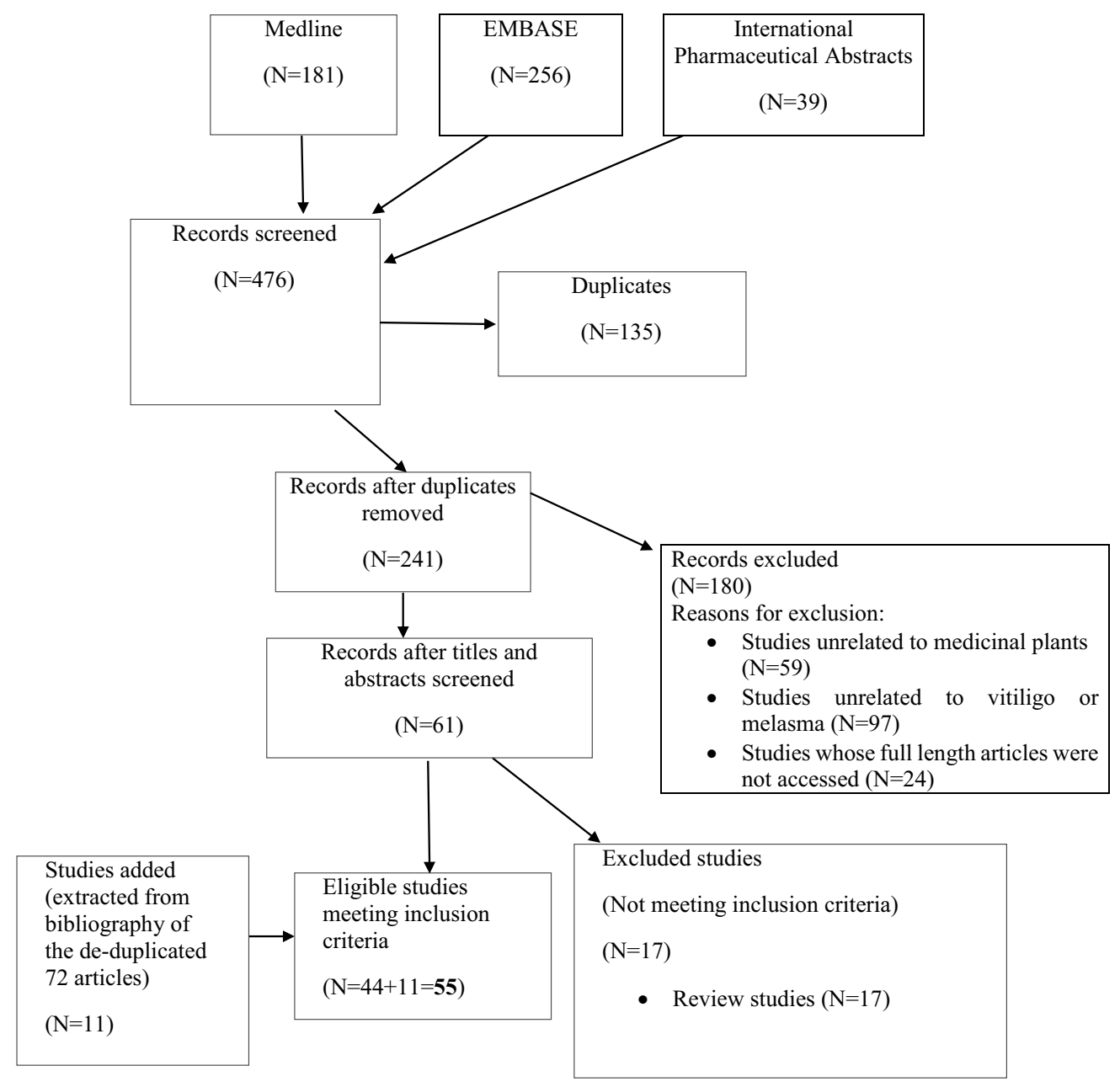

Figure I Flow chart of study selection process.

medicinal plant preparations for the treatment of of vitiligo and hypermelanosis in humans.

\section{Exclusion Criteria}

Publications where full length articles were not available, articles other than English language and articles describing preclinical studies only. Journals discontinued/articles not found online from journal archives.

\section{Results}

The results have been summarized in Tables 3, Tables 2-4.

\section{Discussions}

The study included all types of clinical trials and case reports retrieved according to protocol and the literature search strategy. It was found that adverse effects were poorly reported in most of the studies. Some research papers mentioned safety in abstract but did not provide details, some trials did not report safety information, some did report, but not very well. The topical gel Aloe vera was reported safe without any significant dermatological reactions by Masoumech and $\mathrm{Ali}^{7}$ in a randomized double blind controlled trial. On Aloe vera VigiAccess reported 204 cases of total adverse events including 29 reports on skin and subcutaneous reactions and 1 report on pigmentation disorders specifically the discoloration of skin. The report about discoloring/hypopigmentation effect of Aloe vera pointed out the use of Aloe vera in skin care and anti-aging effects. Debbie ${ }^{9}$ worked on topical lotion of Coffea Arabica; fruit, vegetables extract in hyperpigmentation and and reported no side effects. The study was on a limited number of patients (40 females) for a short period of time ( 5 weeks). Hussain et $\mathrm{al}^{11}$ reported skin irritation effects with topical ointment contained powdered form of Psoralea corylifolia (PC). Maurice and 


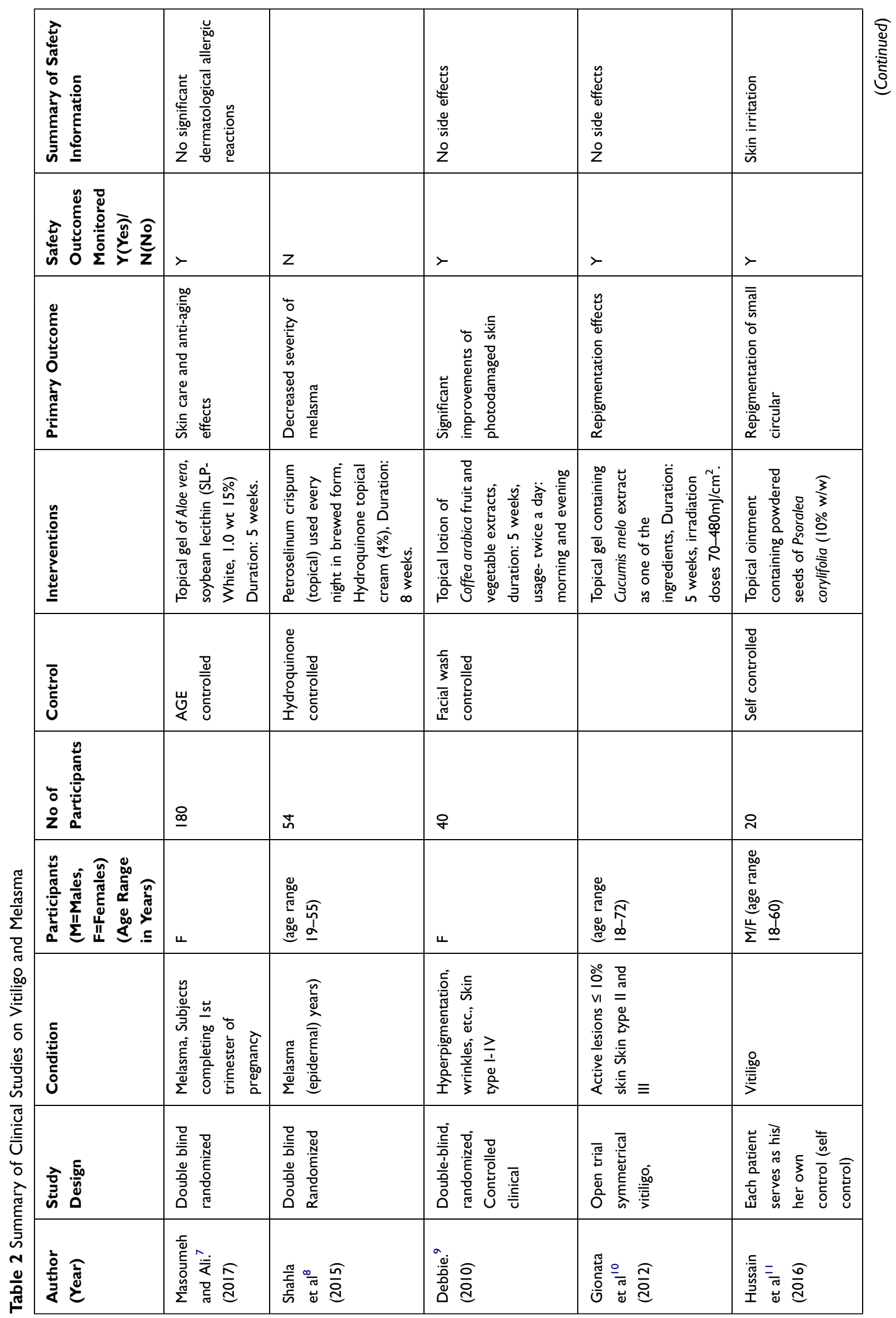




\begin{tabular}{|c|c|c|c|c|}
\hline 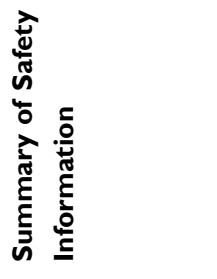 & 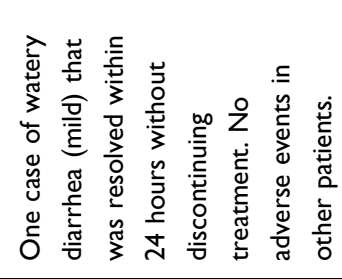 & 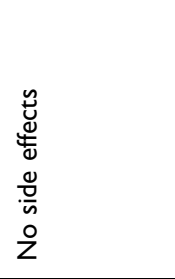 & 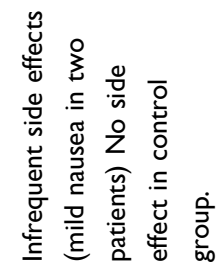 & 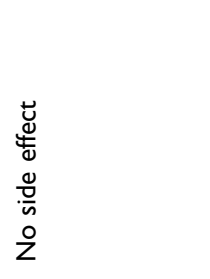 \\
\hline 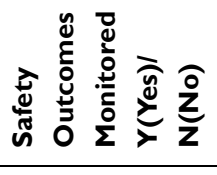 & $\succ$ & $\succ$ & $\succ$ & $\succ$ \\
\hline 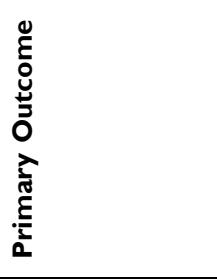 & 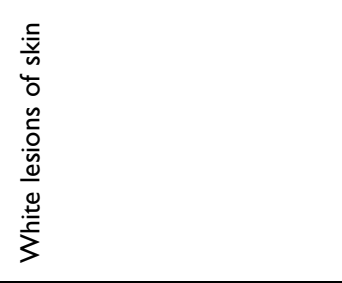 & 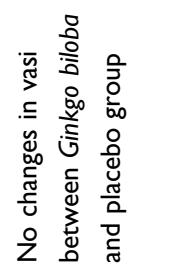 & 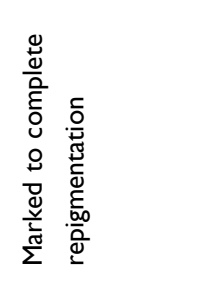 & 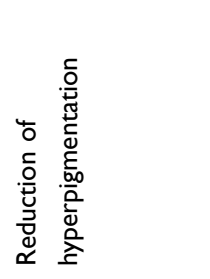 \\
\hline 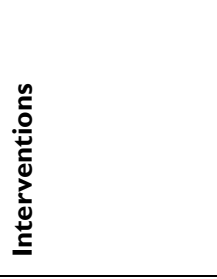 & 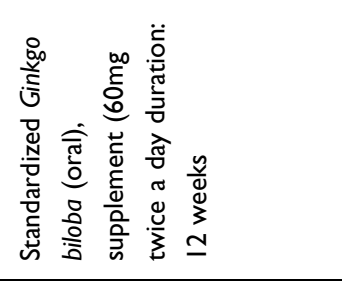 & 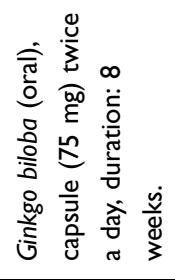 & 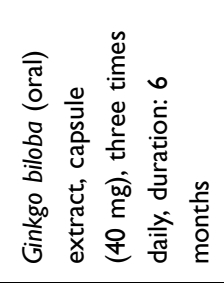 & 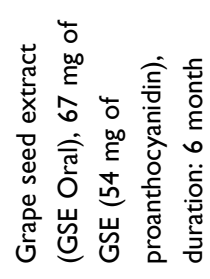 \\
\hline 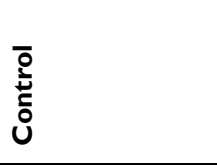 & $\begin{array}{l}\bar{o} \\
\stackrel{\bar{t}}{0} \\
\overline{0} \\
\text { o } \\
\end{array}$ & 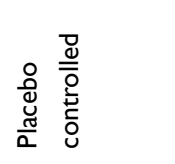 & 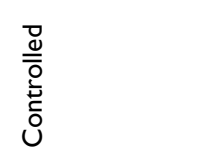 & 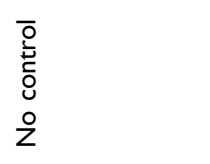 \\
\hline 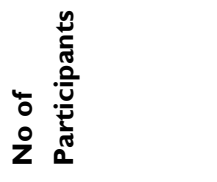 & $\simeq$ & in & กิ & $\simeq$ \\
\hline 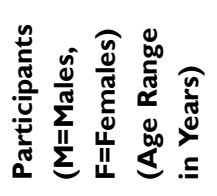 & 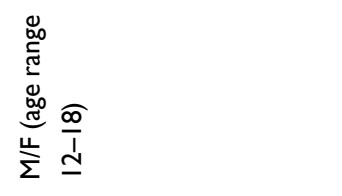 & 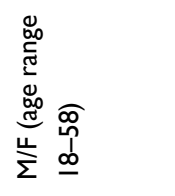 & $\frac{\omega}{\Sigma}$ & ч \\
\hline 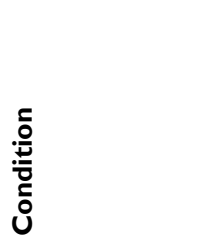 & 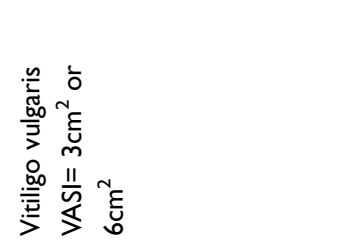 & 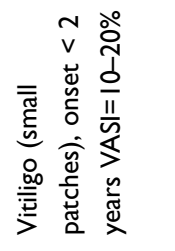 & 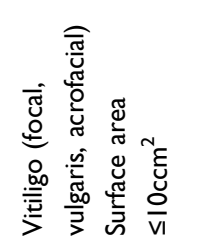 & 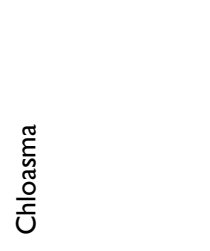 \\
\hline 窟 & 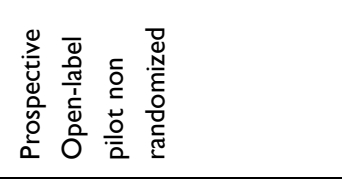 & 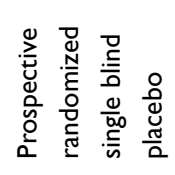 & 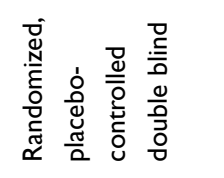 & 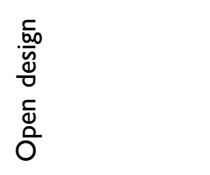 \\
\hline 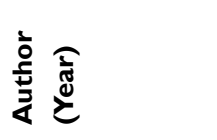 & $\begin{array}{lll}\ddot{y} & \frac{\pi}{\pi} & \bar{D} \\
\dot{0} & \stackrel{0}{d}\end{array}$ & 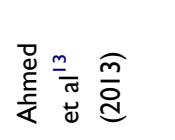 & 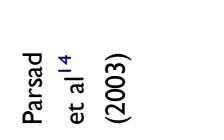 & 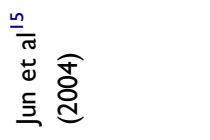 \\
\hline
\end{tabular}




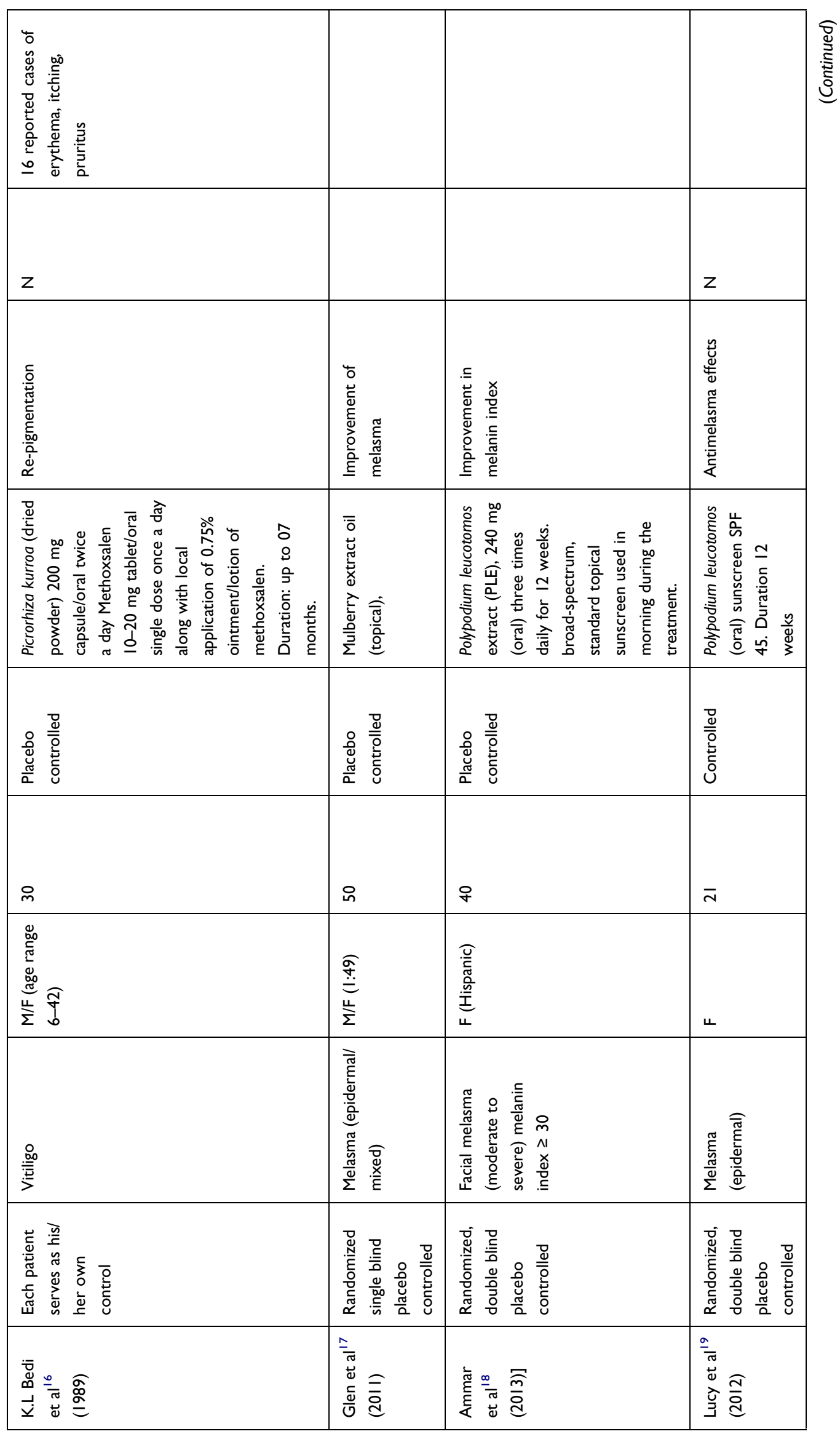




\begin{tabular}{|c|c|c|c|}
\hline 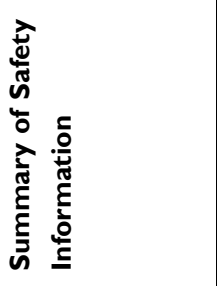 & & 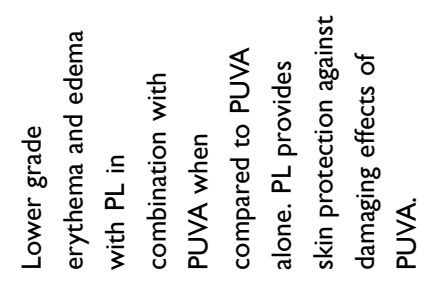 & \\
\hline 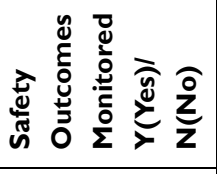 & $\succ$ & $\succ$ & $\succ$ \\
\hline 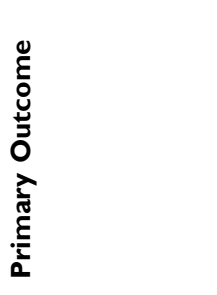 & 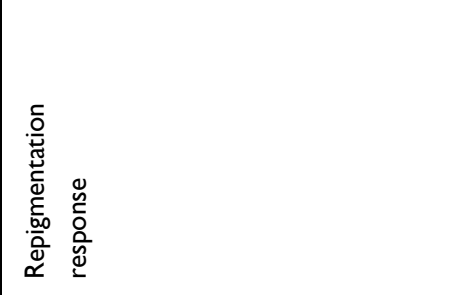 & 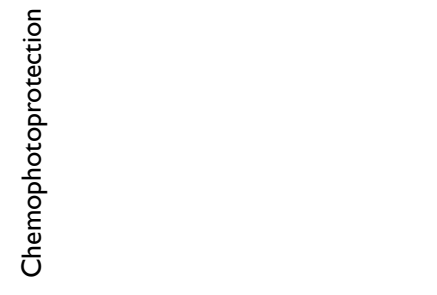 & 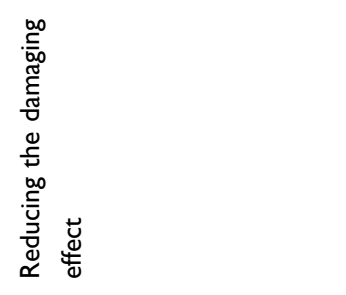 \\
\hline & 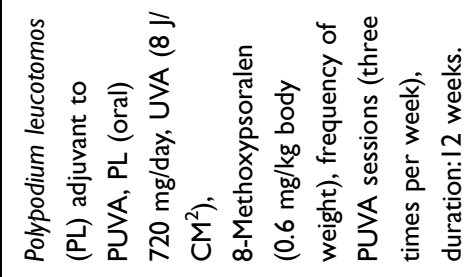 & 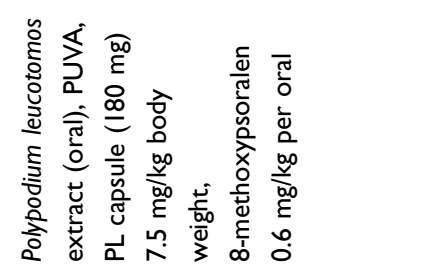 & 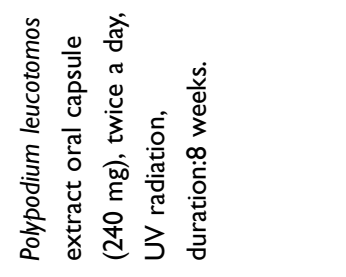 \\
\hline 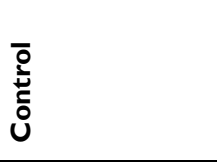 & & 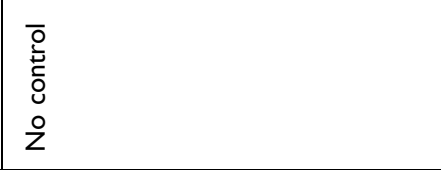 & 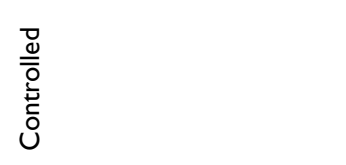 \\
\hline 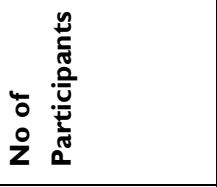 & $\underline{\sigma}$ & $\circ$ & o \\
\hline 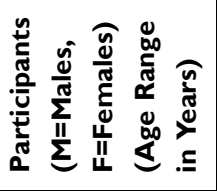 & 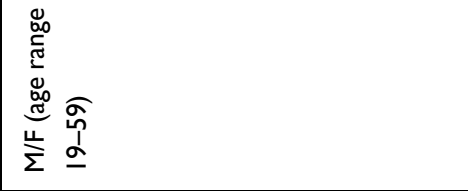 & 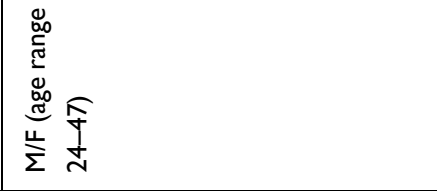 & 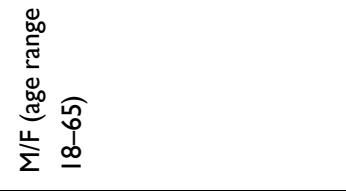 \\
\hline 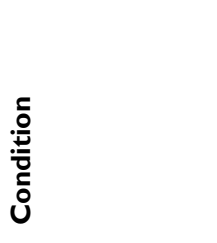 & 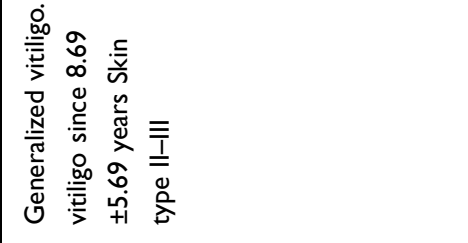 & 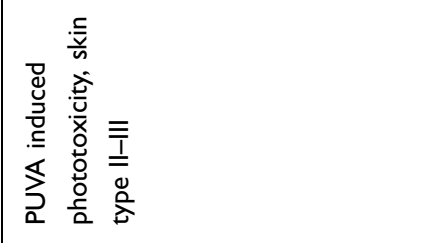 & 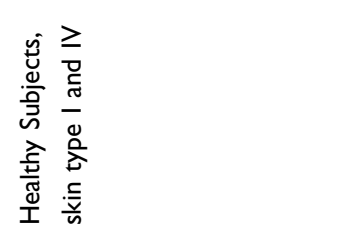 \\
\hline 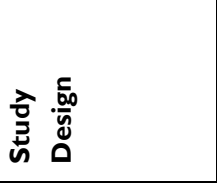 & 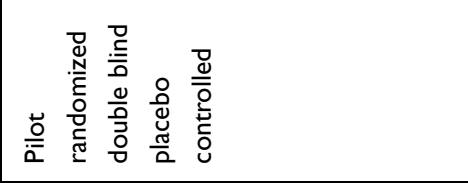 & 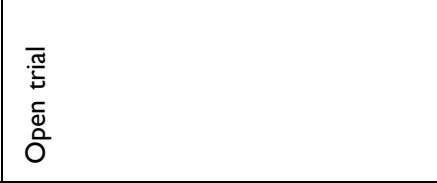 & 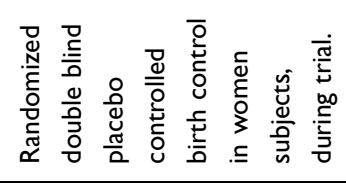 \\
\hline 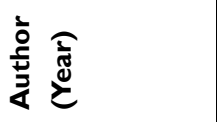 & 莡용 & 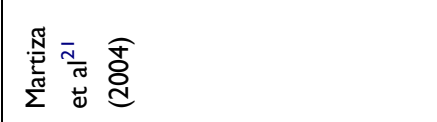 & 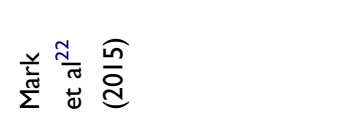 \\
\hline
\end{tabular}




\begin{tabular}{|c|c|c|c|c|}
\hline & 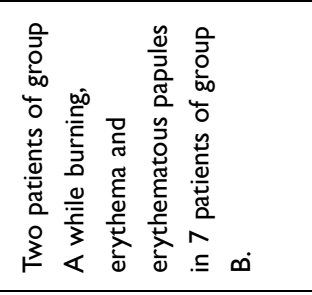 & 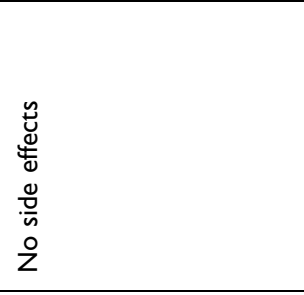 & 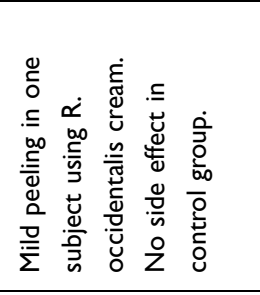 & 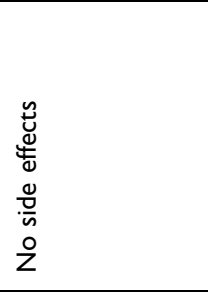 \\
\hline$\succ$ & $\succ$ & $\succ$ & > & $\succ$ \\
\hline 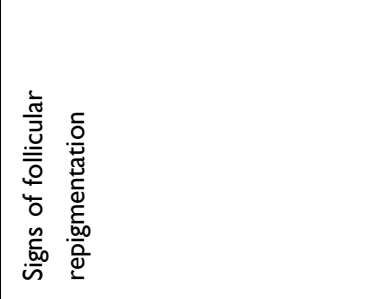 & 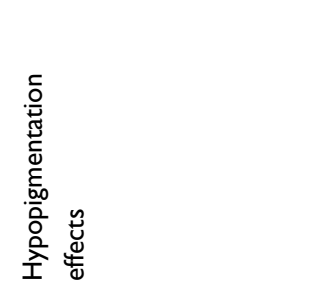 & & 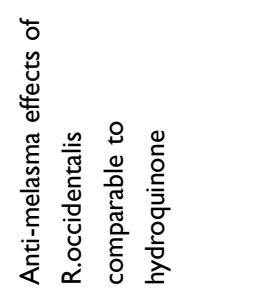 & 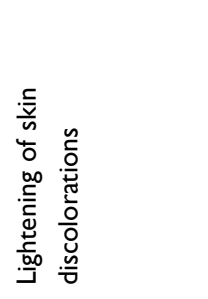 \\
\hline 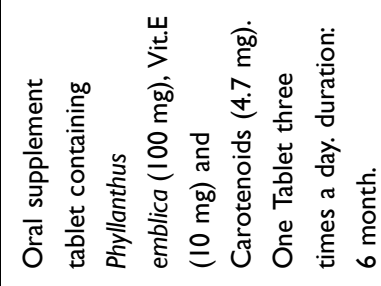 & 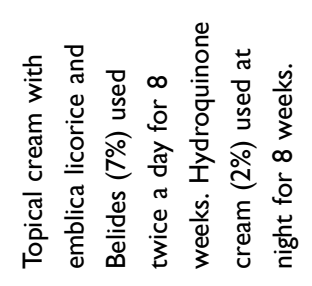 & 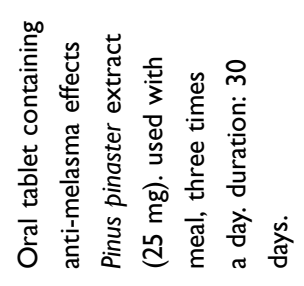 & 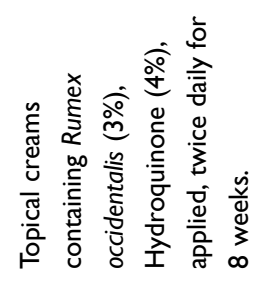 & 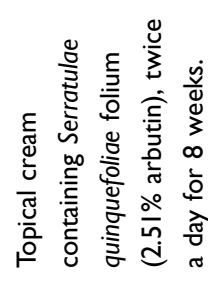 \\
\hline $\begin{array}{l}\overline{\bar{g}} \\
\overline{\underline{z}} \\
\overline{0} \\
\dot{0}\end{array}$ & 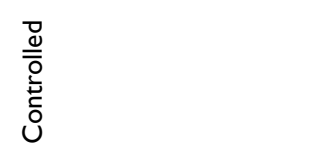 & $\begin{array}{l}\overline{\mathrm{g}} \\
\overline{\mathrm{z}} \\
\mathrm{o} \\
\mathrm{o} \\
\mathrm{z}\end{array}$ & 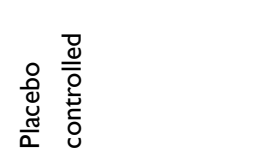 & 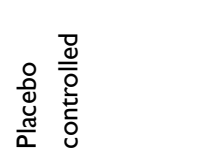 \\
\hline$\stackrel{\circ}{m}$ & & m & ஜ & $\underline{\Xi}$ \\
\hline 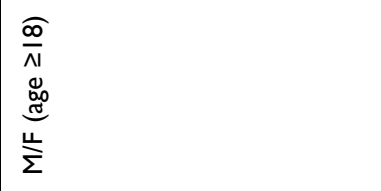 & 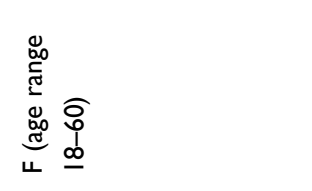 & 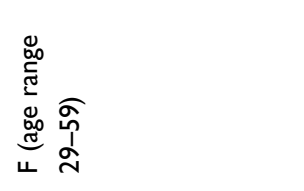 & 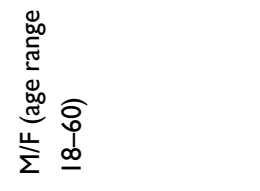 & 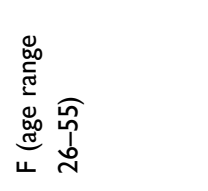 \\
\hline 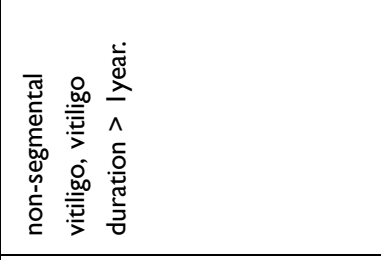 & 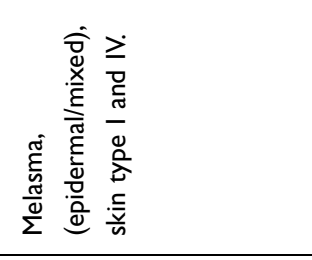 & $\begin{array}{l}\frac{\tilde{5}}{5} \\
\frac{\tilde{m}}{\tilde{\omega}} \\
\end{array}$ & 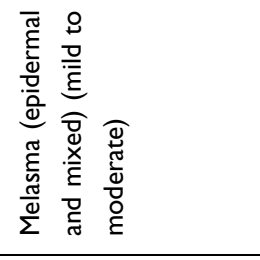 & 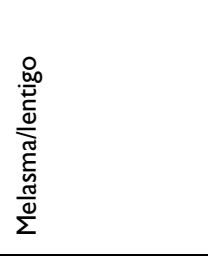 \\
\hline 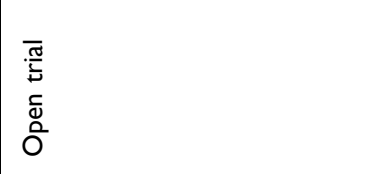 & 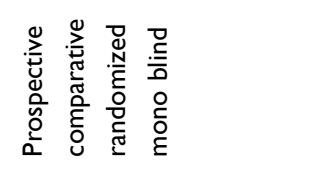 & 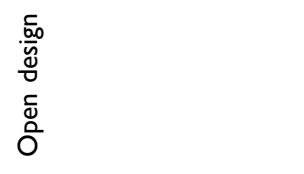 & 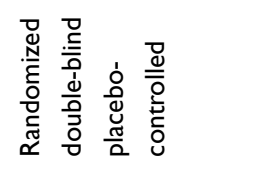 & 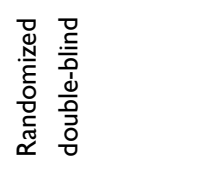 \\
\hline 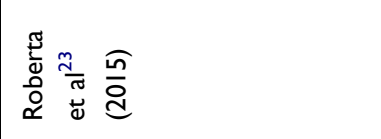 & 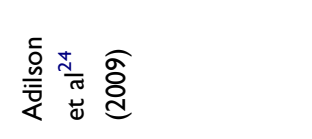 & 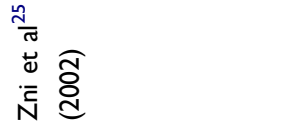 & 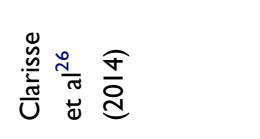 & 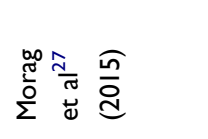 \\
\hline
\end{tabular}




\begin{tabular}{|c|c|c|c|}
\hline 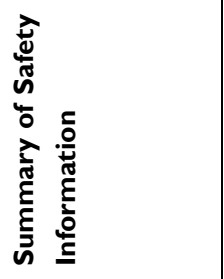 & & 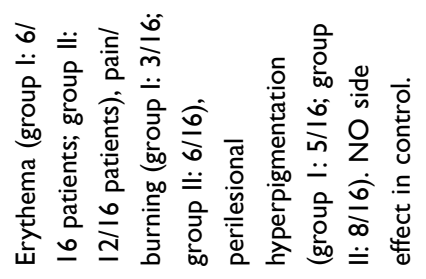 & 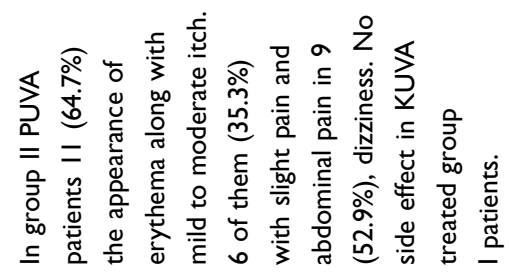 \\
\hline 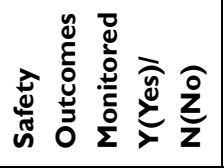 & $z$ & $\succ$ & $\succ$ \\
\hline 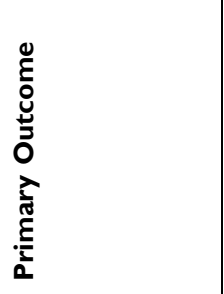 & 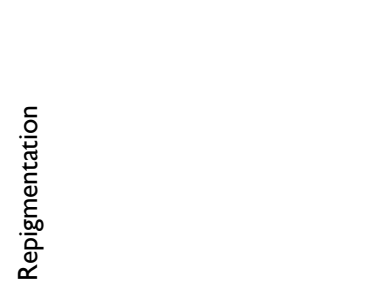 & 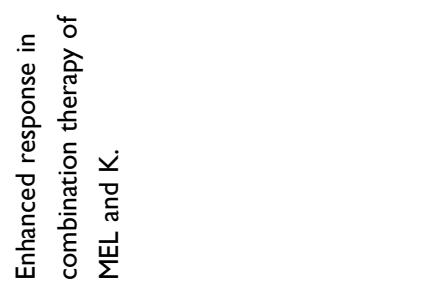 & 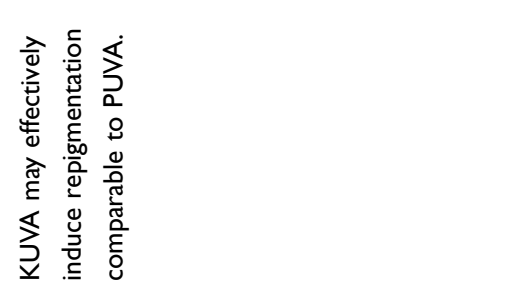 \\
\hline 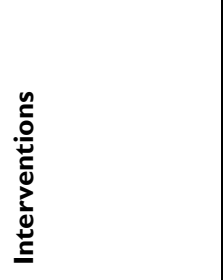 & 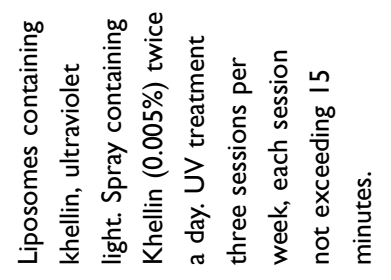 & 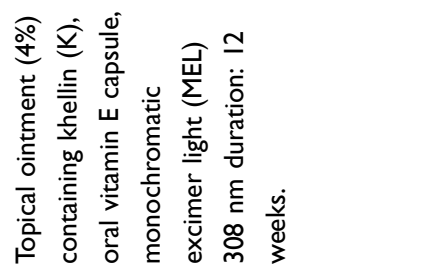 & 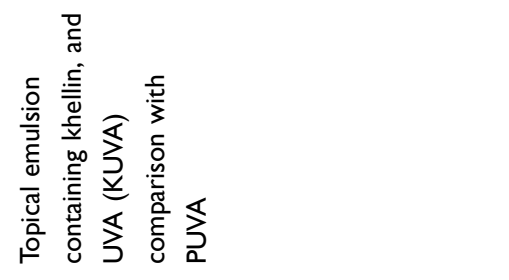 \\
\hline 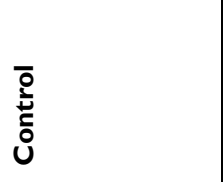 & $\begin{array}{l}\overline{0} \\
\stackrel{0}{2} \\
0 \\
0 \\
\text { ò }\end{array}$ & 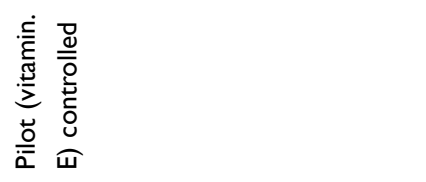 & 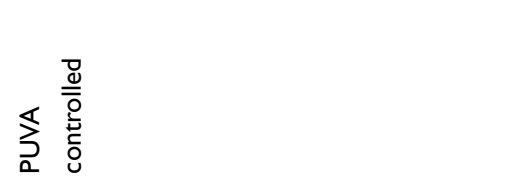 \\
\hline zo & $\underline{a}$ & $\stackrel{\infty}{+}$ & $\tilde{m}$ \\
\hline 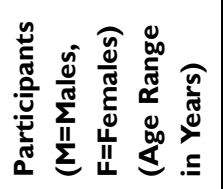 & 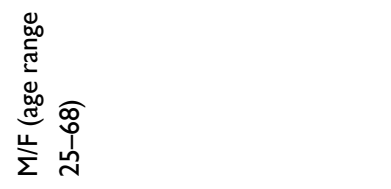 & 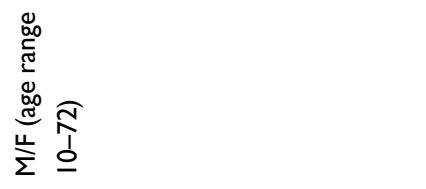 & 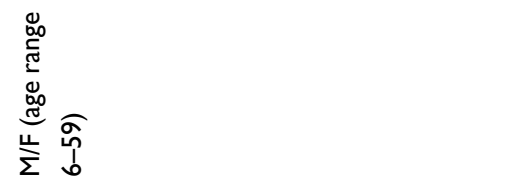 \\
\hline 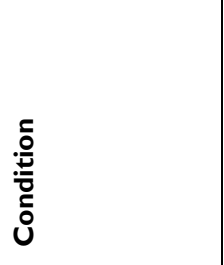 & 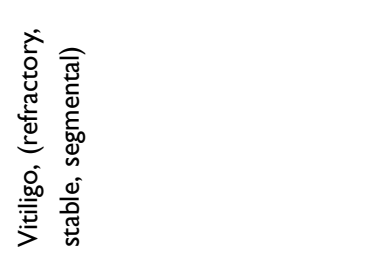 & 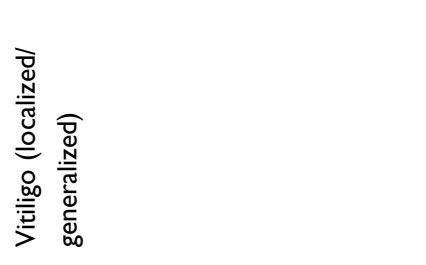 & 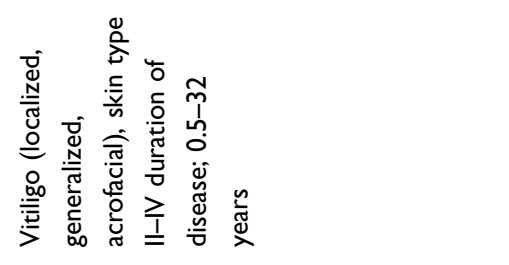 \\
\hline 宕高离 & 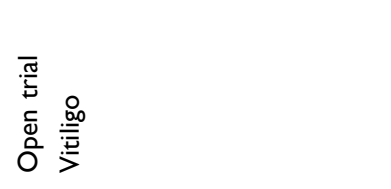 & 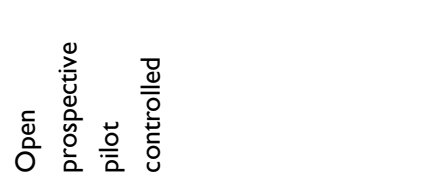 & 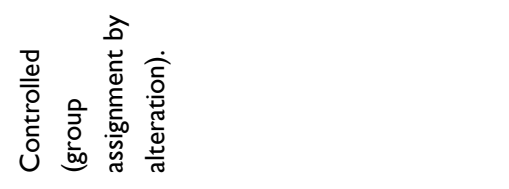 \\
\hline 辛 & 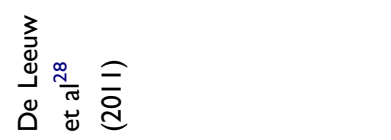 & 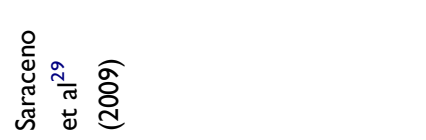 & 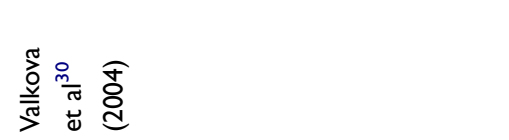 \\
\hline
\end{tabular}




\begin{tabular}{|c|c|c|}
\hline 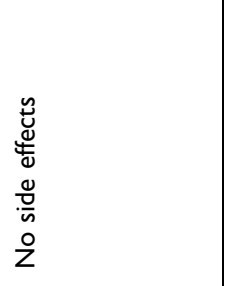 & 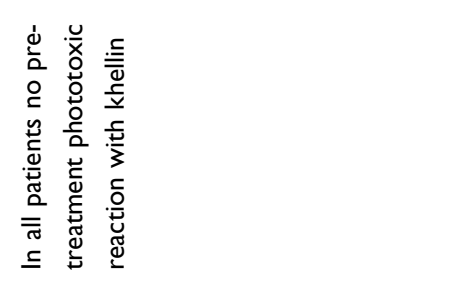 & 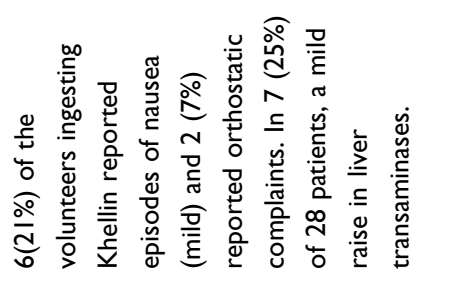 \\
\hline$\nu$ & 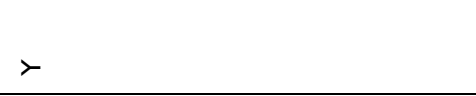 & $>$ \\
\hline 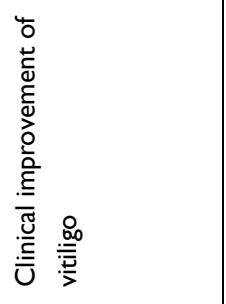 & 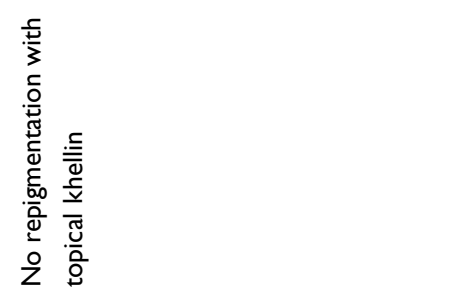 & 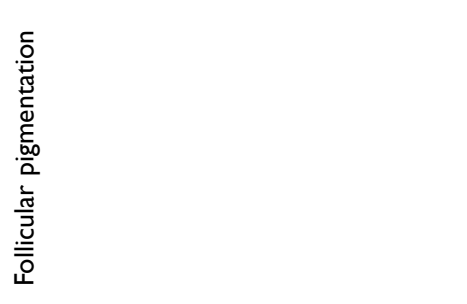 \\
\hline 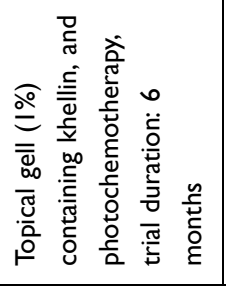 & 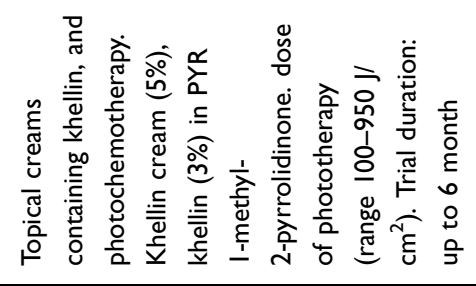 & 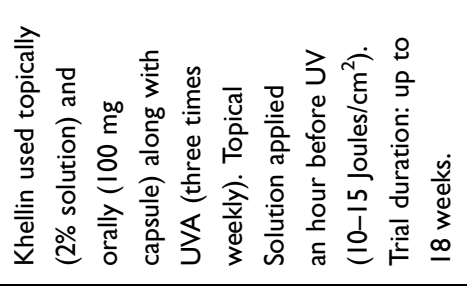 \\
\hline 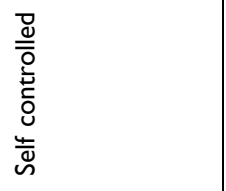 & 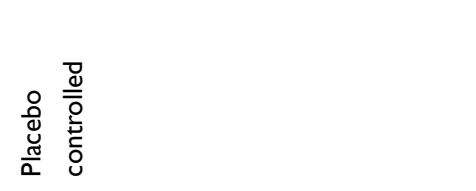 & 容 \\
\hline m & N & $\stackrel{\infty}{\sim}$ \\
\hline 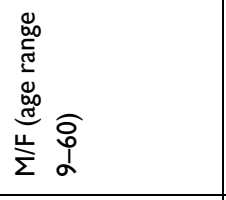 & 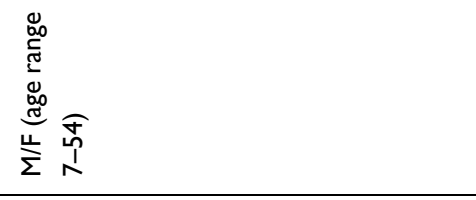 & 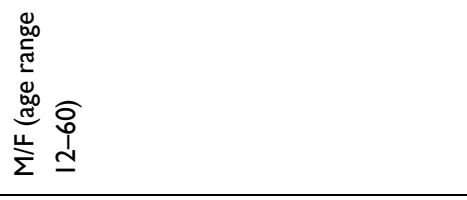 \\
\hline 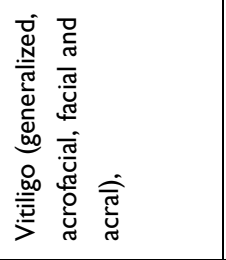 & 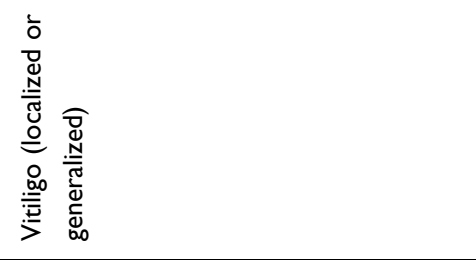 & 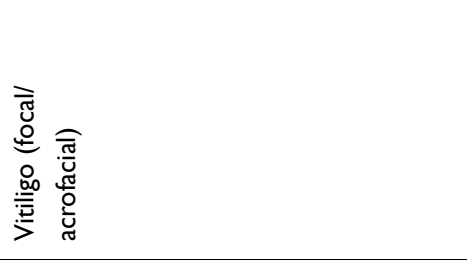 \\
\hline 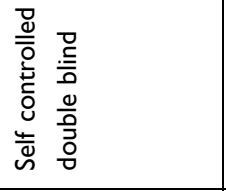 & 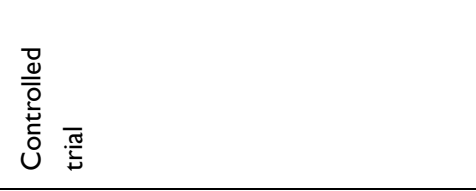 & 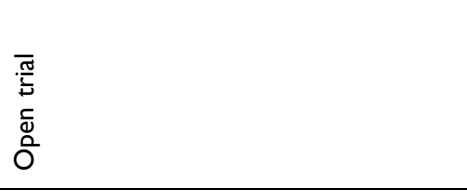 \\
\hline 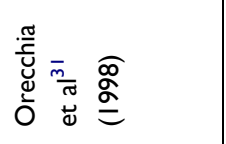 & 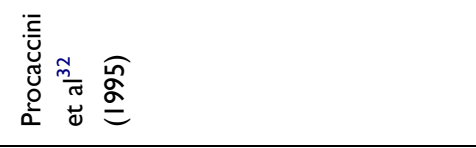 & 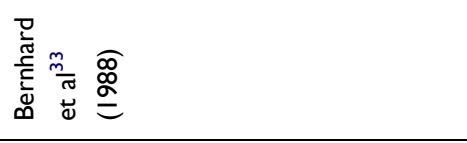 \\
\hline
\end{tabular}




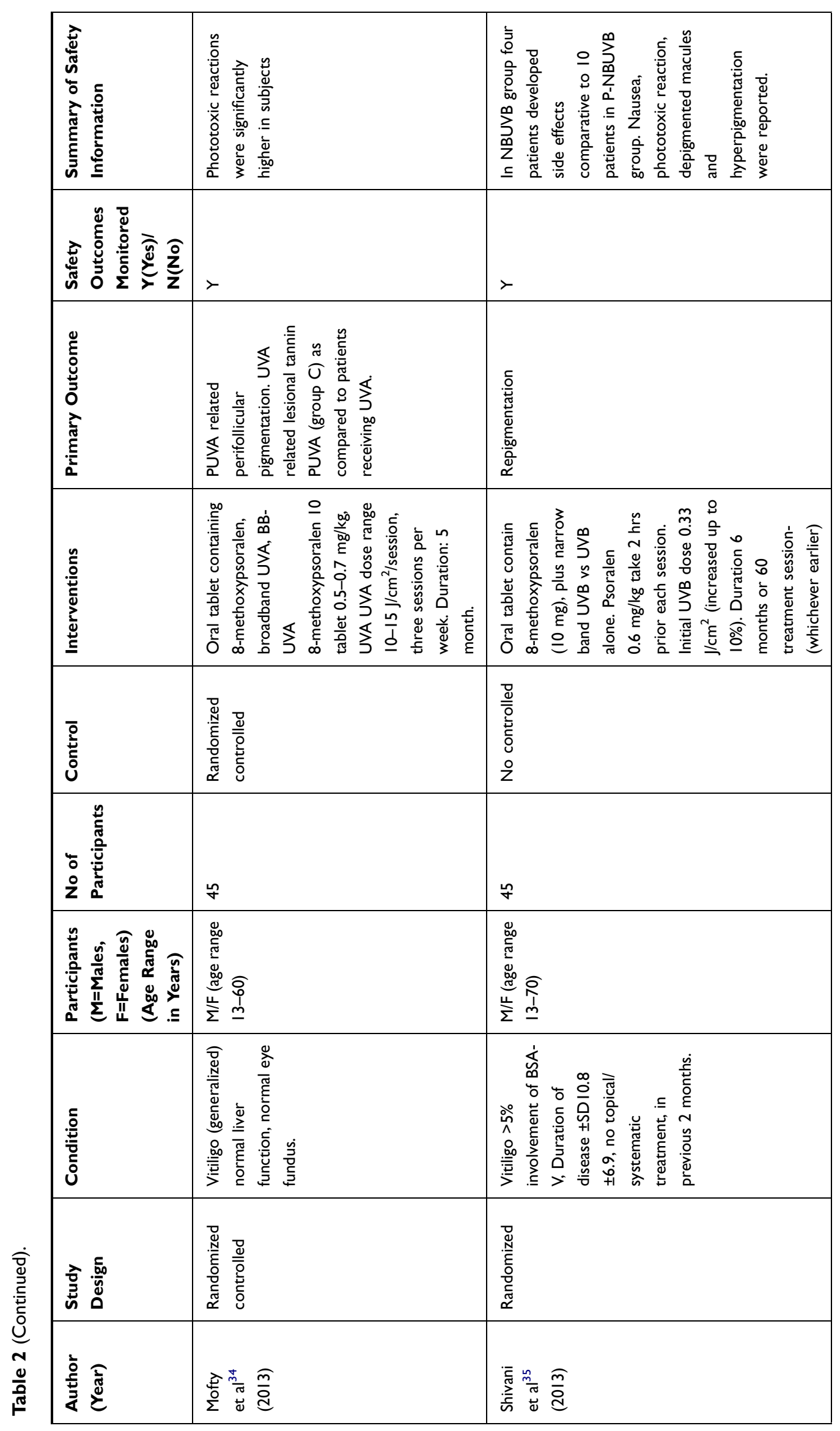




\begin{tabular}{|c|c|}
\hline 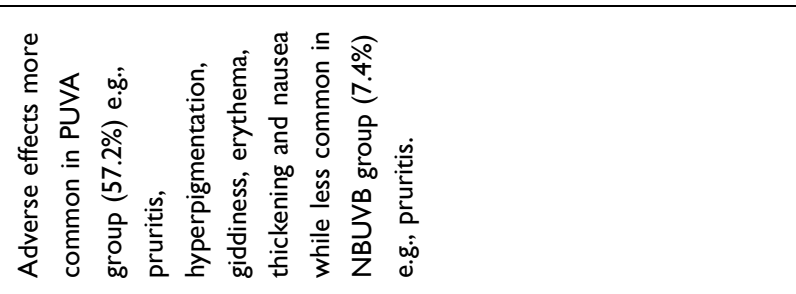 & 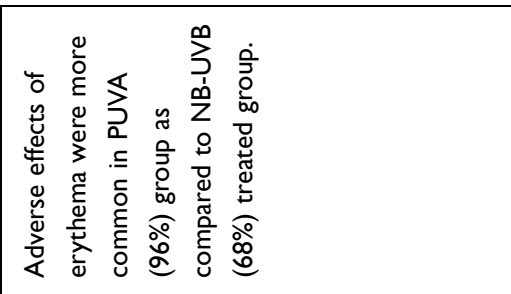 \\
\hline$\nu$ & $\succ$ \\
\hline 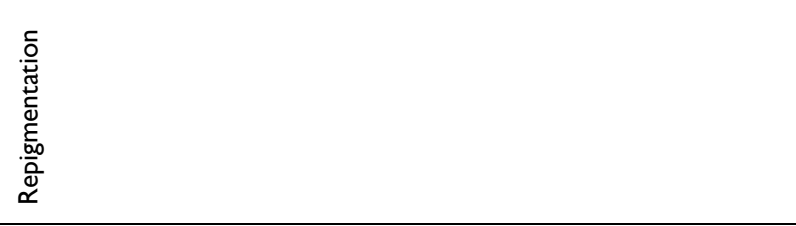 & 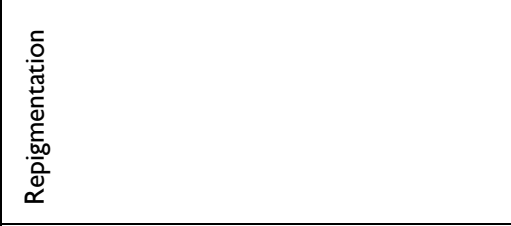 \\
\hline 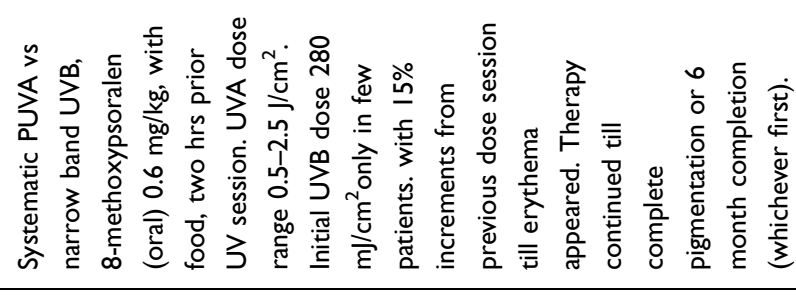 & 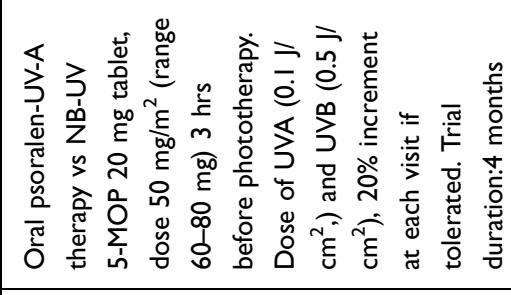 \\
\hline 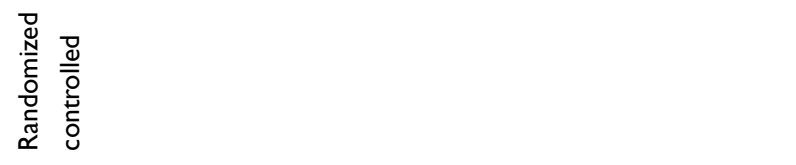 & 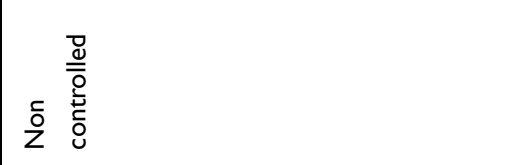 \\
\hline i̊ & 茼 \\
\hline 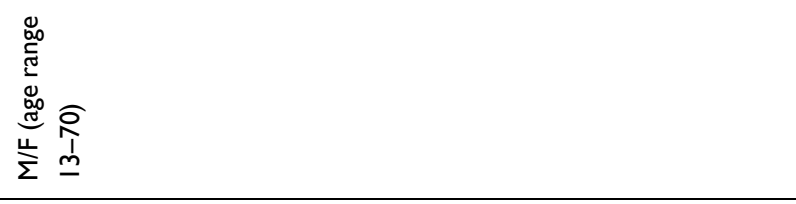 & 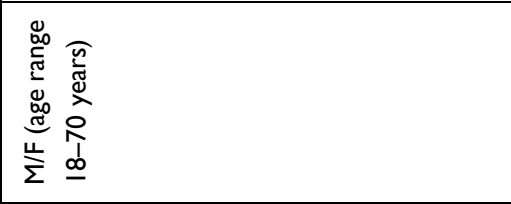 \\
\hline 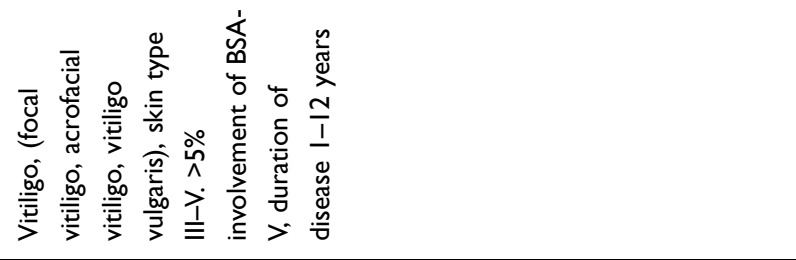 & 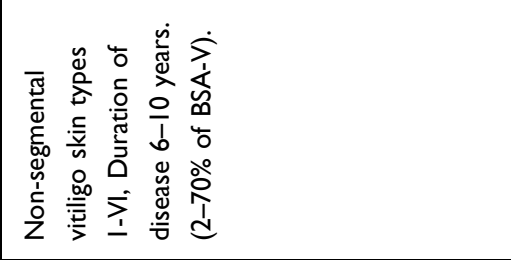 \\
\hline 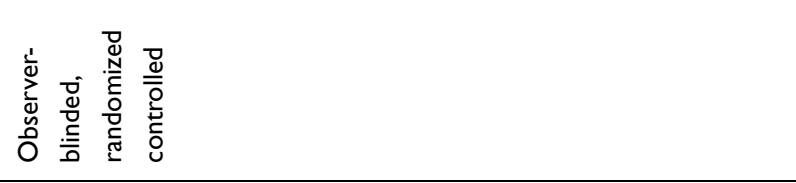 & 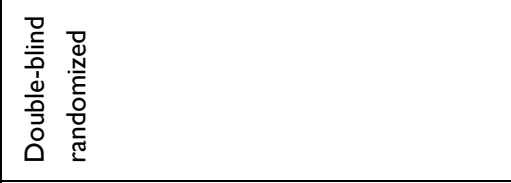 \\
\hline 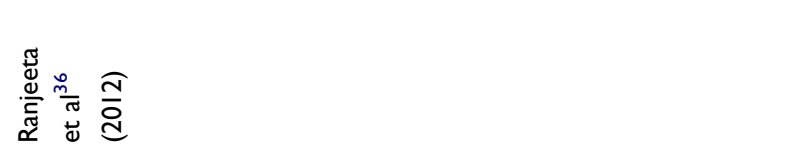 & 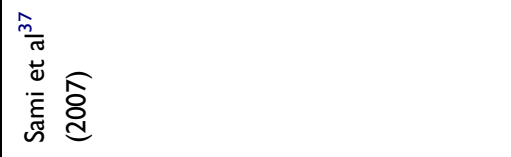 \\
\hline
\end{tabular}




\begin{tabular}{|c|c|c|c|}
\hline 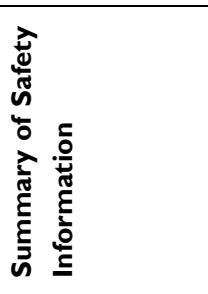 & & 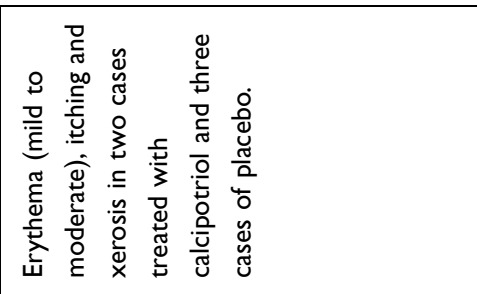 & 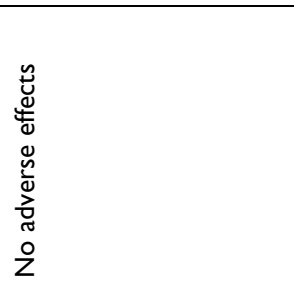 \\
\hline 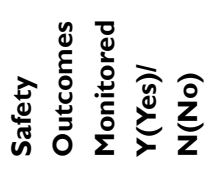 & $z$ & $\succ$ & $\succ$ \\
\hline 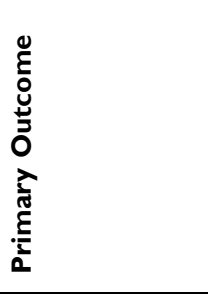 & 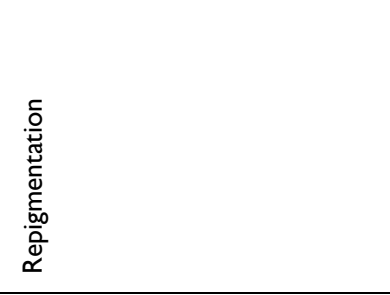 & 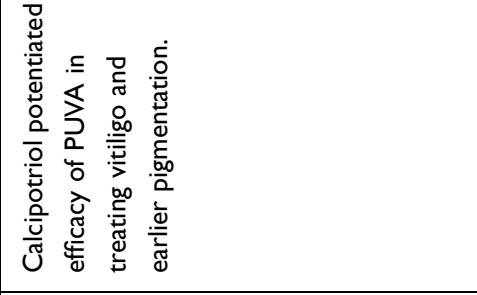 & 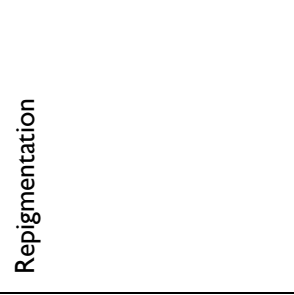 \\
\hline 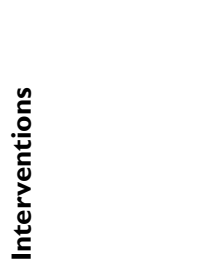 & 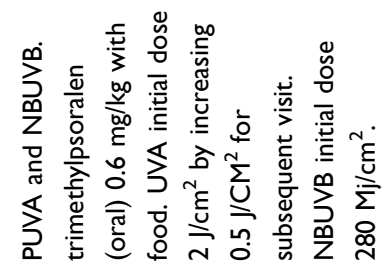 & 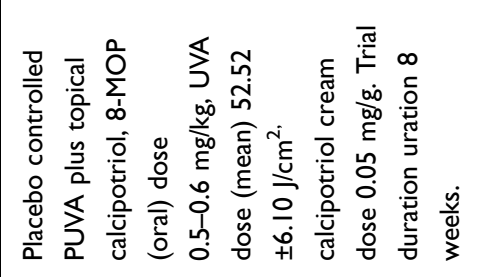 & 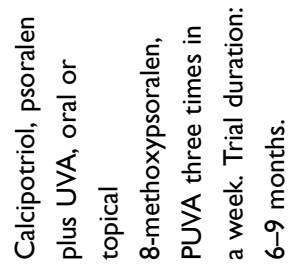 \\
\hline סे & 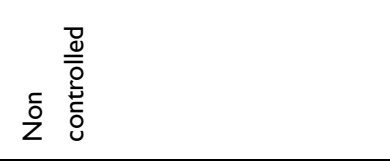 & & 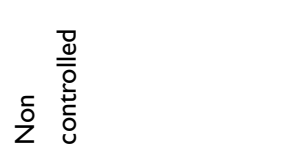 \\
\hline 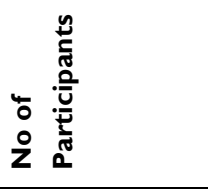 & in & $\stackrel{\sim}{m}$ & $\stackrel{\text { న }}{2}$ \\
\hline 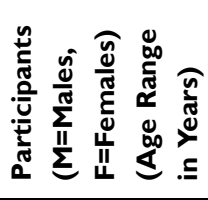 & 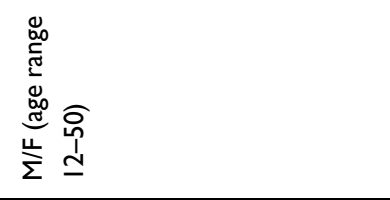 & 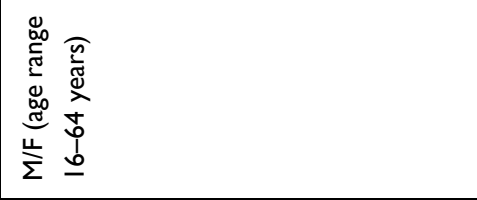 & 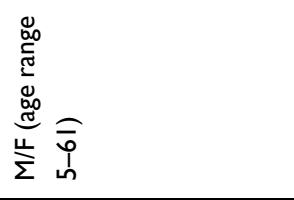 \\
\hline 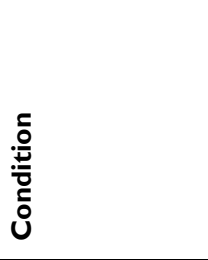 & 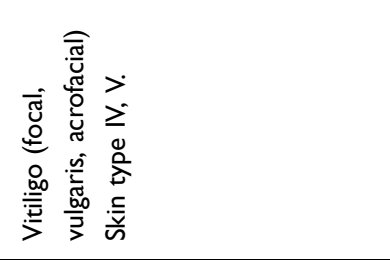 & 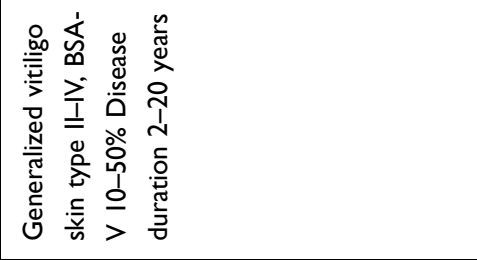 & 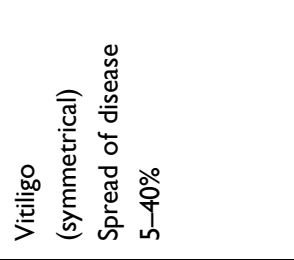 \\
\hline 窟点 & 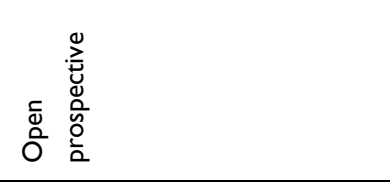 & 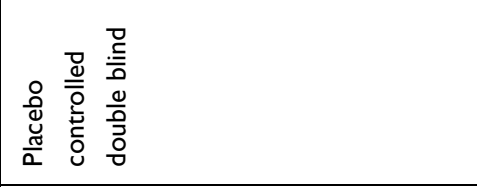 & 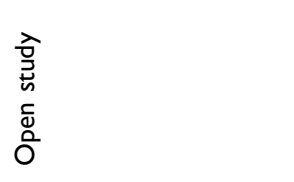 \\
\hline 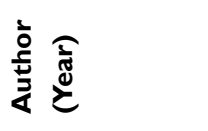 & 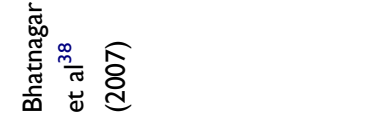 & 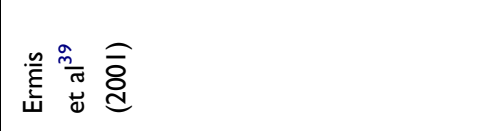 & 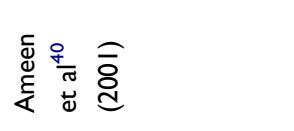 \\
\hline
\end{tabular}




\begin{tabular}{|c|c|c|c|}
\hline & & 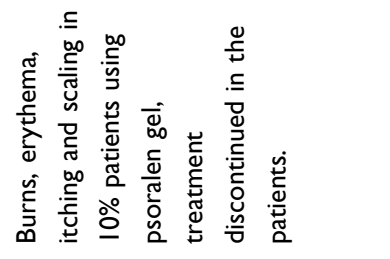 & 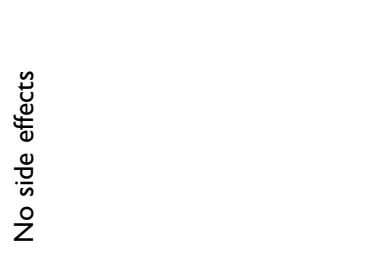 \\
\hline$z$ & $z$ & $\succ$ & $\succ$ \\
\hline 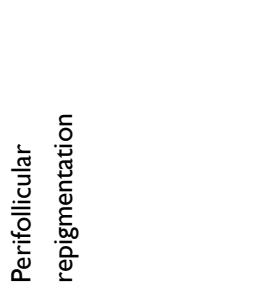 & 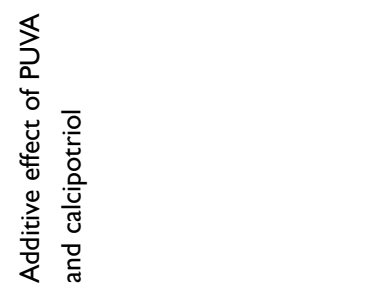 & 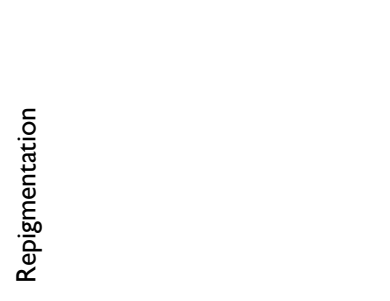 & 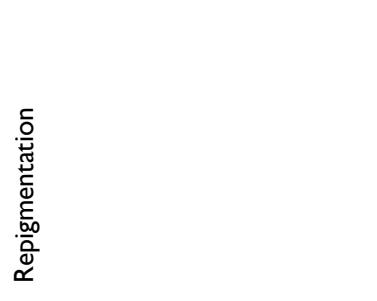 \\
\hline 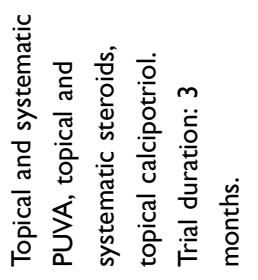 & 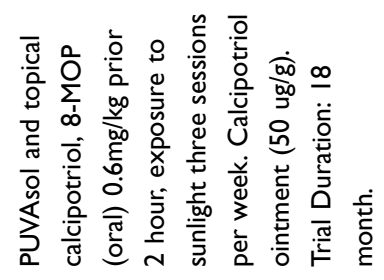 & 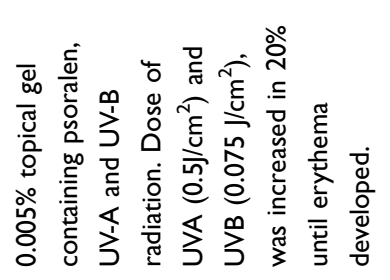 & 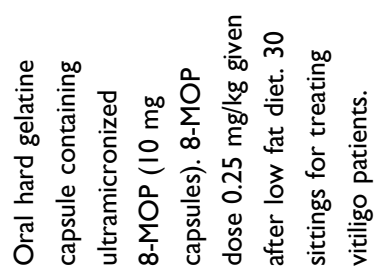 \\
\hline 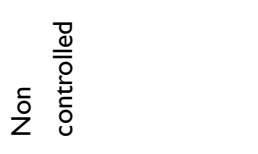 & 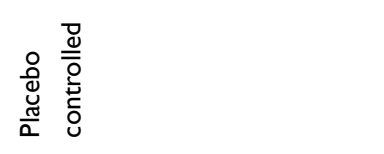 & 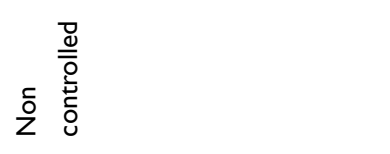 & 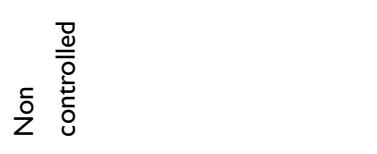 \\
\hline$\stackrel{\Perp}{\simeq}$ & $\underline{a}$ & $\underline{\bar{\infty}}$ & กิ \\
\hline 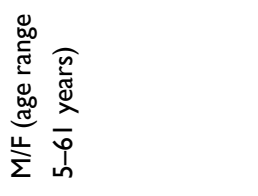 & 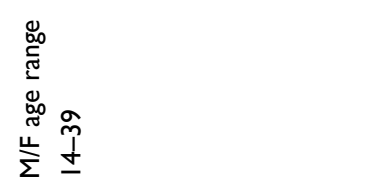 & $\frac{u}{\Sigma}$ & 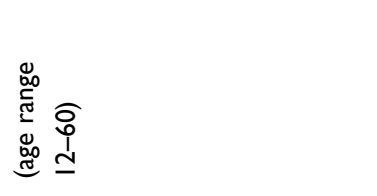 \\
\hline 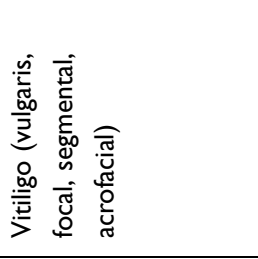 & 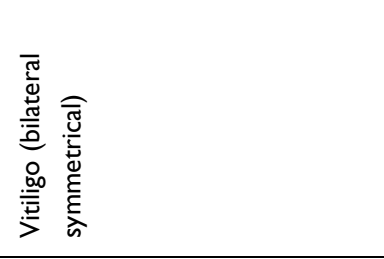 & 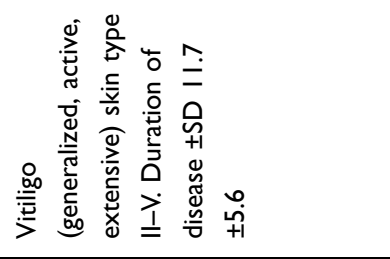 & 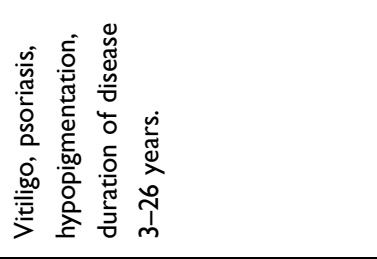 \\
\hline 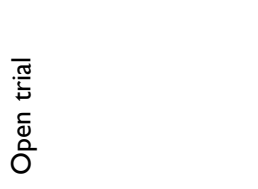 & 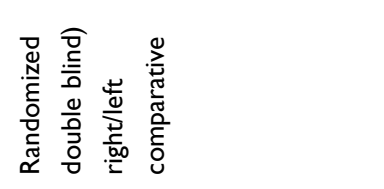 & 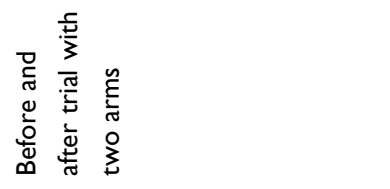 & 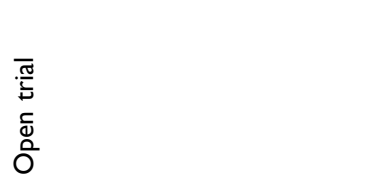 \\
\hline 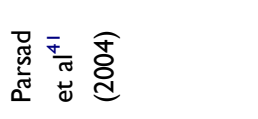 & 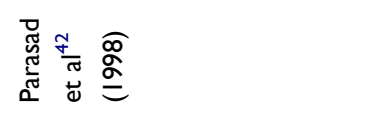 & 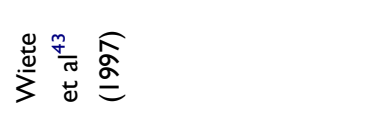 & 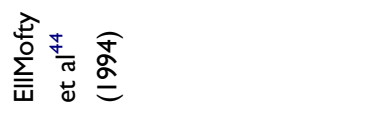 \\
\hline
\end{tabular}




\begin{tabular}{|c|c|c|}
\hline 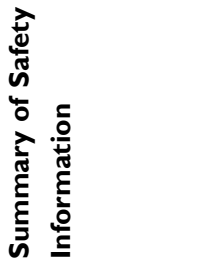 & 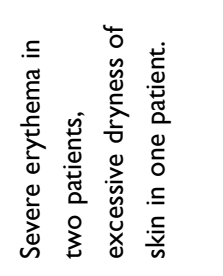 & 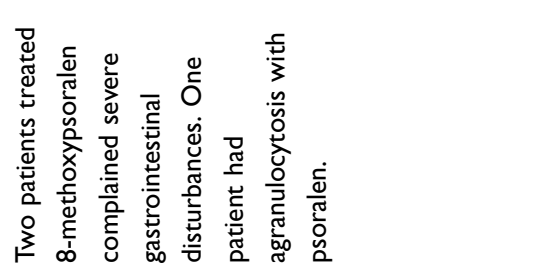 \\
\hline 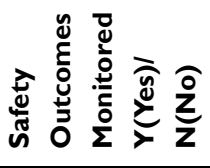 & $\succ$ & $\succ$ \\
\hline 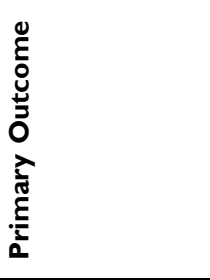 & 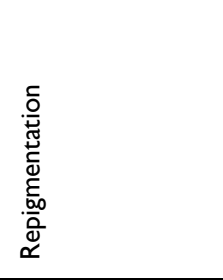 & 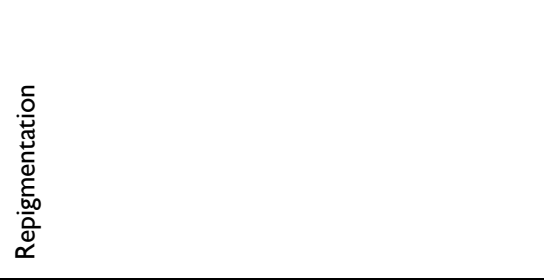 \\
\hline 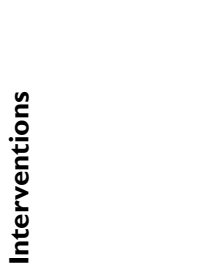 & 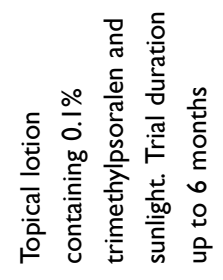 & 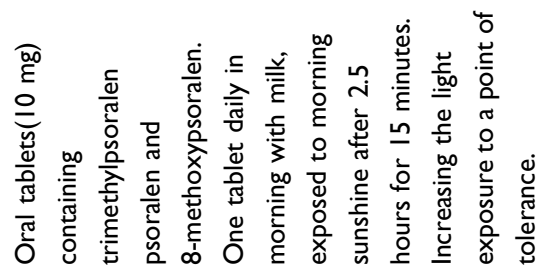 \\
\hline 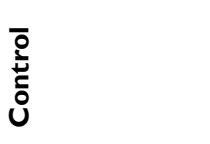 & z & 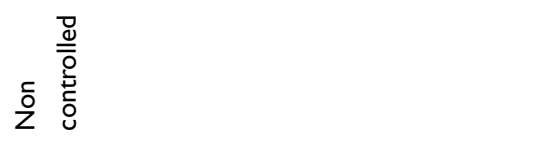 \\
\hline 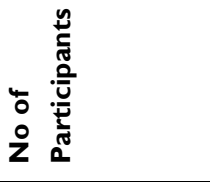 & $\stackrel{ \pm}{\sim}$ & $\hat{\infty}$ \\
\hline 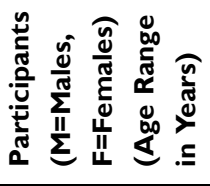 & $\frac{\mathrm{U}}{\Sigma}$ & $\frac{\omega}{\Sigma}$ \\
\hline ن̃ & 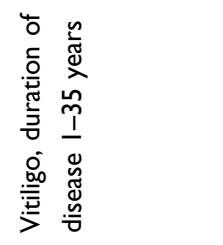 & $\frac{.00}{\frac{.00}{\bar{E}}}$ \\
\hline 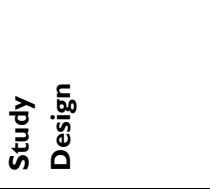 & 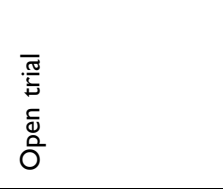 & 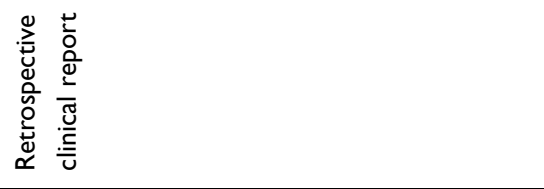 \\
\hline 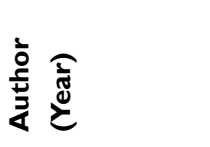 & 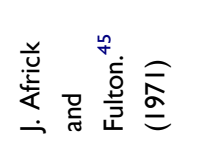 & 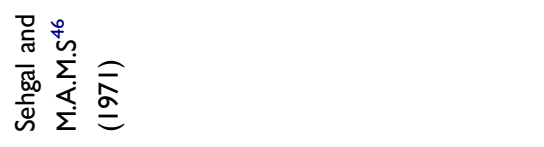 \\
\hline
\end{tabular}




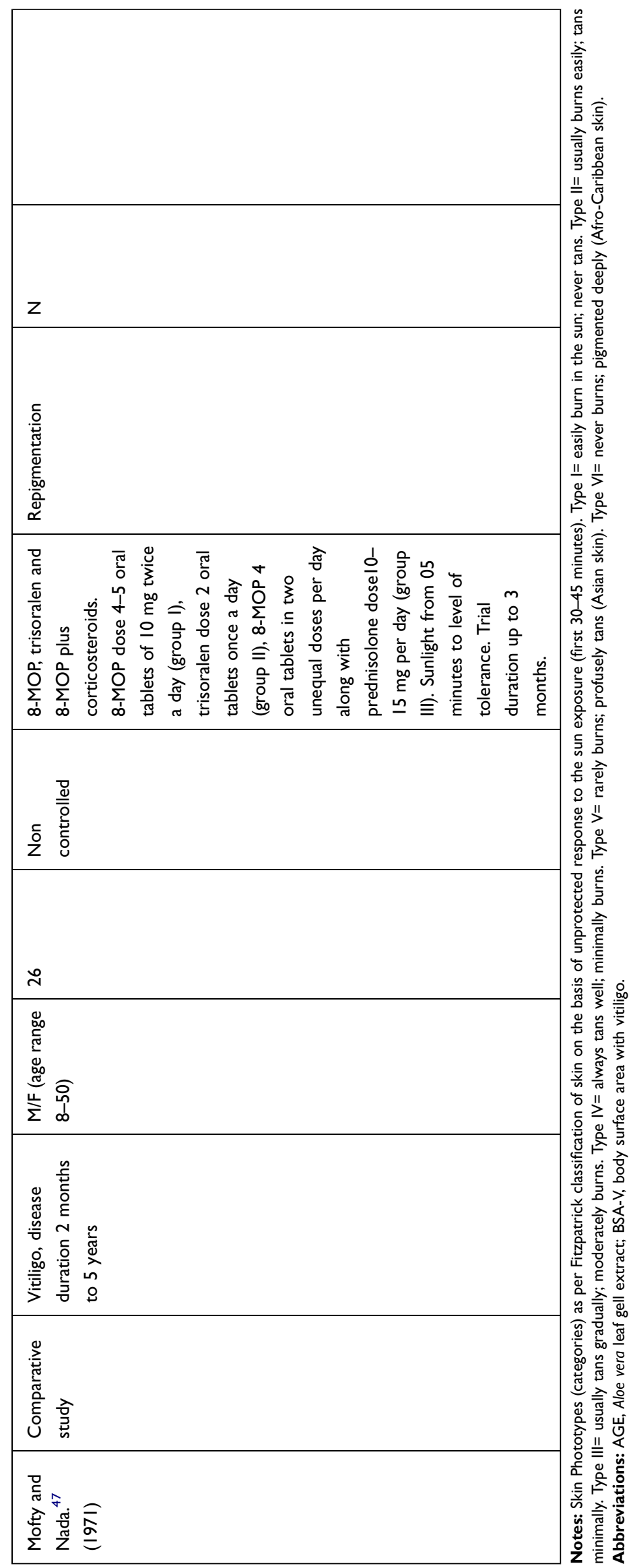


Table 3 Summary of Clinical Case Reports on Vitiligo and Melasma

\begin{tabular}{|c|c|c|c|c|c|}
\hline $\begin{array}{l}\text { Author } \\
\text { (Year) }\end{array}$ & $\begin{array}{l}\text { Patient } \\
\text { Characteristics } \\
\text { (Age, Sex) }\end{array}$ & $\begin{array}{l}\text { Details of the Condition } \\
\text { Treated }\end{array}$ & Details of Medicines Used & Suspected ADRs & Outcome \\
\hline $\begin{array}{l}\text { Peter et al }{ }^{48} \\
(200 I)\end{array}$ & 48 years, female & Vitiligo for ten years & $\begin{array}{l}\text { I Khellin ( } 100 \mathrm{mg} / \text { oral) } 2.5 \text { hours } \\
\left.\text { before UVA ( } 10 \text { joules } / \mathrm{cm}^{2}\right) \\
\text { three times a week. 2) } 2 \% \\
\text { khellin solution (topical) }\end{array}$ & $\begin{array}{l}\text { A marked } \\
\text { elevation in LFTs } \\
\text { with oral and } \\
\text { topical therapy. } \\
\text { slight } \\
\text { hepatomegaly }\end{array}$ & $\begin{array}{l}\text { Liver functions } \\
\text { returned to normal } \\
\text { after the treatment } \\
\text { stopped. }\end{array}$ \\
\hline $\begin{array}{l}\text { A Kreuter } \\
\text { et } \mathrm{al}^{49}\end{array}$ & 39 years, female & $\begin{array}{l}\text { Cosmetically disturbing } \\
\text { extensive exacerbated } \\
\text { depigmented lesions on face. }\end{array}$ & $\begin{array}{l}\text { Topical glucocorticoids, topical } \\
\text { cream containing psoralen and } \\
\text { UVA }\left(0.5 \mathrm{~J} / \mathrm{cm}^{2}-4 \mathrm{~J} / \mathrm{cm}^{2} \text {. }\right.\end{array}$ & No side effects & $\begin{array}{l}\text { Complete } \\
\text { repigmentation after } \\
80 \text { treatment sessions }\end{array}$ \\
\hline $\begin{array}{l}\text { Maurice and } \\
\text { Cream }^{50} \\
(1989)\end{array}$ & 30 years, male & $\begin{array}{l}\text { Extensive vitiligo for twenty } \\
\text { years, photosensitivity, itching of } \\
\text { hands. }\end{array}$ & $\begin{array}{l}\text { Herbal infusion containing } \\
\text { powdered seeds of Psoralea } \\
\text { corylifolia in a daily dose } \\
\text { equivalent to } 30 \mathrm{~g} \text { of seeds. }\end{array}$ & $\begin{array}{l}\text { Photosensitivity, } \\
\text { itching, of hands, } \\
\text { erythema, } \\
\text { blistering. }\end{array}$ & $\begin{array}{l}\text { Three month after } \\
\text { stop of taking herbal } \\
\text { infusion ADRs were } \\
\text { reversed. }\end{array}$ \\
\hline $\begin{array}{l}\text { David et } \mathrm{al}^{51} \\
\text { (1998) }\end{array}$ & 9 years, female & $\begin{array}{l}\text { Progressive vitiligo involving } \\
\text { axillae, lower extremities and } \\
\text { trunk BSA }=10 \%\end{array}$ & $\begin{array}{l}\text { Bath PUVA, 8-MOP }(0.0002 \%) \text {. } \\
\text { Initial dose of UVA } 0.25 \mathrm{~J} / \mathrm{cm}^{2} \\
\text { with increment of } 0.25 \mathrm{~J} / \mathrm{cm}^{2} \\
\text { after every other treatment. } \\
\text { Maximum UVA dose } 3.0 \mathrm{~J} / \mathrm{cm}^{2}\end{array}$ & No side effects & $\begin{array}{l}\text { Nearly complete re- } \\
\text { pigmentation of } \\
\text { vitiliginous areas. }\end{array}$ \\
\hline $\begin{array}{l}\text { Zouhir } \\
\text { Djerrou. }^{52} \\
(2015)\end{array}$ & 34 years, male & $\begin{array}{l}\text { Localized vitiligo on face and } \\
\text { neck, diabetes and thyroid } \\
\text { dysfunction. }\end{array}$ & $\begin{array}{l}\text { History of using conventional } \\
\text { drugs. natural remedy formed } \\
\text { from honey bee, decoction of } \\
\text { dry oat stems and red onion } \\
\text { juice taken every evening daily. } \\
\text { Exposure to sunlight for I5-20 } \\
\text { minutes/day. Citrus lemon fruit } \\
\text { juice (limited use). }\end{array}$ & No side effects & $\begin{array}{l}\text { Complete re- } \\
\text { pigmentation }\end{array}$ \\
\hline $\begin{array}{l}\text { Rigmor }^{53} \\
(1976)\end{array}$ & 40 years, female & $\begin{array}{l}\text { Vitiligo since } 14 \text { years of age, } \\
\text { symmetrically localized vitiligo } \\
\text { over trunk, back of feet and } \\
\text { wrists. Achlorhydria, pernicious } \\
\text { anemia diabetes mellitus and } \\
\text { thyroid gland diseases. }\end{array}$ & $\begin{array}{l}\text { Methoxsalen }(20-30 \mathrm{mg} / \text { oral }) \\
\text { and sunlight for } 91 \text { days. History } \\
\text { of taking arsenic trioxide } \\
(1000 \mathrm{mg}) .\end{array}$ & $\begin{array}{l}\text { Moderate } \\
\text { erythema of skin, } \\
\text { multiple basal cell } \\
\text { carcinomas. }\end{array}$ & $\begin{array}{l}\text { Raised Risk of skin } \\
\text { cancer with } \\
\text { methoxsalen and sun } \\
\text { light in the patient } \\
\text { with history of using } \\
\text { carcinogenics. }\end{array}$ \\
\hline $\begin{array}{l}\text { Deborah and } \\
\text { MacDonald } \\
\text { (20I4) }\end{array}$ & 52 years, female & Vitiligo & $\begin{array}{l}\text { Oral use of seeds of Psoralea } \\
\text { corylifolia }\end{array}$ & $\begin{array}{l}\text { Cholestatic acute } \\
\text { hepatitis, jaundice, } \\
\text { abdominal pain, } \\
\text { lethargy, vomiting, } \\
\text { dark urine, pale } \\
\text { stools. }\end{array}$ & $\begin{array}{l}\text { Reversal of acute } \\
\text { hepatitis on stoppage } \\
\text { of using seeds of } \\
\text { Psoralea corylifolia. }\end{array}$ \\
\hline $\begin{array}{l}\text { M.B. Abdel } \\
\text { et } \mathrm{al}^{55} \text { (2004) }\end{array}$ & 22 years, female & Generalized vitiligo & $\begin{array}{l}\text { Systemic PUVA therapy } \\
\text { (cumulative dose } 1750 \mathrm{~J} / \mathrm{cm}^{2} \text { ) }\end{array}$ & $\begin{array}{l}\text { PUVA induced } \\
\text { lentigines }\end{array}$ & $\begin{array}{l}\text { Appearance of } \\
\text { lentigines in in } \\
\text { addition to } \\
\text { pigmentation of skin. }\end{array}$ \\
\hline
\end{tabular}

Cream, ${ }^{50}$ a case report concerning the use of herbal infusion containing powdered seeds of Psoralea corylifolia, mentioned the adverse effects of photosensitivity, itching of hands, erythema, and blistering. The reversal of ADR after stoppage of the infusion it was related to the use of PC. Deborah and MacDonald ${ }^{54}$ reported 
Table 4 Brief Summary of Adverse Events from VigiAccess ${ }^{56}$

\begin{tabular}{|c|c|c|c|c|}
\hline $\begin{array}{l}\text { Name of Medicinal } \\
\text { Plants }\end{array}$ & $\begin{array}{l}\text { Total No. of } \\
\text { Reports }\end{array}$ & $\begin{array}{l}\text { Skin + Subcutaneous Tissue } \\
\text { Disorders (No. of Reports) }\end{array}$ & $\begin{array}{l}\text { Reports Related to } \\
\text { Pigmentation Disorders }\end{array}$ & $\begin{array}{l}\text { Details (Where } \\
\text { Applicable) }\end{array}$ \\
\hline $\begin{array}{l}\text { Centella asiatica Gotu } \\
\text { kola }\end{array}$ & 122 & 46 & 0 & \\
\hline Artemisia capillaris & I & 0 & 0 & \\
\hline Fallopia multiflora & 20 & 0 & 0 & \\
\hline Eclipta prostrata & 5 & 0 & 0 & \\
\hline Rehmannia glutinosa & 36 & 2 & 0 & \\
\hline $\begin{array}{l}\text { Hippophae rhamnoides } \\
\text { sea buckthorn }\end{array}$ & 3 & 1 & 0 & \\
\hline Cassia fistula & I & 0 & 0 & \\
\hline $\begin{array}{l}\text { Psoralea corylifolia cullen } \\
\text { corylifolium }\end{array}$ & 16 & 3 & 0 & \\
\hline Zingiber officinale Gine & 108 & 41 & 0 & \\
\hline Piper nigrum & 2 & 0 & & \\
\hline Piper longum & I & 0 & 0 & \\
\hline $\begin{array}{l}\text { Petroselinum crispum } \\
\text { parsley }\end{array}$ & 25 & 0 & 0 & \\
\hline Ammi visnaga & 16 & 2 & 0 & \\
\hline Khellin & 7 & 2 & 0 & \\
\hline Ammi visnaga & 16 & 2 & & \\
\hline Ginkgo bilobo & 4016 & 66 & 7 & Skin discoloration \\
\hline Morus alba & 7 & 0 & 0 & \\
\hline Aloe vera & 204 & 29 & I & Skin discoloration \\
\hline $\begin{array}{l}\text { Withania somnifera } \\
\text { ashwagandha }\end{array}$ & 54 & 7 & 0 & \\
\hline Glechoma hederacea & 13 & 0 & 0 & \\
\hline Achillea millefolium & 4 & 0 & 0 & \\
\hline Ricinus communis & 147 & 24 & 0 & \\
\hline Vitis vinifera & 1729 & 293 & 11 & Skin discoloration \\
\hline Pinus pinaster & 7 & 5 & 0 & \\
\hline Juglans regia & II & 2 & 0 & \\
\hline Picrorhiza kurroa & 5 & 0 & 0 & \\
\hline
\end{tabular}

a case of serious ADR with the oral use of PC seeds. urine, and pale stools. Acute hepatitis was reversed The adverse effects were cholestatic acute hepatitis, after stopping the use of seeds of PC. Acute hepatitis jaundice, abdominal pain, lethargy, vomiting, dark was a potential ADR of PC and manufacturers of such 
products should label a warning to highlight this harmful effect of hepatitis. A study recommending no significant side effects of PC did not include the biochemistry of liver function tests, thus hepatitis was unlikely to be observed in this research. ${ }^{57}$ There is need for the proper recommendation of daily dosing of PC as current recommendations are referenced from customs instead of science. ${ }^{58}$ The trials to establish formal dosing is needed. VigiAccess ${ }^{56}$ reported 16 cases of total adverse events with PC including 3 reports on skin and subcutaneous tissue disorders of blister, pruritus and rash. PC is believed to have its local arterial pharmacological action on plexus of the capillaries, dilating them by stimulating melanoblasts to produce pigments. The pigment penetrates in the white vitiliginous patches on skin. ${ }^{59}$ Orest et al, ${ }^{12}$ Ahmed et $\mathrm{al}^{13}$ and Parsad et $\mathrm{al}^{14}$ studied Ginkgo biloba clinically in vitiligo patients. There were infrequent reports on side effects related to gastrointestinal problems. Ginkgo biloba has been widely used in many indications. VigiAccess ${ }^{56}$ reported Ginkgo biloba 4016 cases of total adverse events including 66 reports on skin and subcutaneous reactions and 7 reports related to pigmentation disorders (skin discoloration). Interestingly the information of skin discoloration derived from VigiAccess is opposing the use of Gingko biloba in vitiligo for the purpose of acquiring skin coloration (pigmentation). Patients using Ginkgo biloba are pron to be more risk of increased bleeding. ${ }^{60}$ Bedi et $\mathrm{al}^{16}$ has not monitored the safety of Picrorhiza kurroa in self controlled trials on 30 patients. Some animals studies have reported the drug as potent liver protecting agent to counter the toxicities of poisons by improving the bile flow and rectifying the liver functions. ${ }^{61,62}$ Eduardo $^{20}$ used Polypodium leucotomos (PL) orally adjuvant to PUVA to treat generalized vitiligo, on the other hand, Lucy et $\mathrm{al}^{19}$ tried the same plant alone in the management of melasma. Adverse effects were not monitored by Lucy et $\mathrm{al}^{19}$ while no side effects were reported by Eduardo et al. ${ }^{20}$ Martiza et $\mathrm{al}^{21}$ in an open trial conducted on 10 patients only reported PL plus PUVA as chemophotoprotective along with adverse effects of low grade erythema. Due to its pronounced antioxidative properties PL has protective effect against the UV and PUVA induced damage to skin. It thereby decreases sun burn cells when administered orally or topically to decreases phototoxicity and erythema of psoralens. ${ }^{63,64}$ Colucci et $\mathrm{al}^{23}$ in an adjunct therapy for non-segmental vitiligo reported no side effects for
Phyllanthus emblica when used orally while in another adjunct therapy for melasma reported by Adilson et al, ${ }^{24}$ adverse effects of burning, erythema, and erythematous papules were observed with topical use of the herb. VigiAccesss $^{56}$ reported one adverse event of rash erythematous related to skin and subcutaneous reactions. Khellin has been widely studied as adjunct therapy for its anti-vitiligo effects. Adverse effects reported with adjuvant topical therapy of khellin were erythema, burning sensation and perilesional hyperpigmentation as reported by Saraceno et $\mathrm{al}^{29}$ in the study for vitiligo patients. Whilst no side effects of topical khellin treated patients in vitiligo is observed by Valkova et $\mathrm{al}^{30}$ or Orecchia et al. ${ }^{31}$ Subjects receiving a combination of topical and oral khellin were observed to have episodes of mild nausea, some orthostatic complaints and mild derangement of liver transaminases. A case reported by Dushet et $\mathrm{al}^{48}$ regarding oral plus topical administration of khellin, showed that there was marked elevation of liver transaminases along with slight hepatomegaly. The liver functions were reversed to normal after the treatment was stopped thus confirming the khellin-related ADR. It has been reported previously that use of khellin can raise the transaminases in the first two months of treatment. ${ }^{65}$ The dramatic increase in values of liver enzymes was not observed in a trial on 60 patients. It was surprising that the increase in liver transaminases was reported by Dushet et $\mathrm{al}^{48}$ with topical application of the khellin although systematic absorption as well as serum level of the drug was not monitored. One cannot expect the systematic absorption of the drug merely due to its topical application on vitiliginous lesions. ${ }^{66}$ No liver toxicity was reported with several years' use of Khellin with a daily dosage of up to $300 \mathrm{mg}$ in treatment of cardio patients. ${ }^{67}$ Although some later studies have mentioned the elevation of liver enzymes with the use of Khellin plus UVA. ${ }^{68}$ The results of KUVA are comparable to the findings reported with the use of PUVA but the major advantages of khellin were no side effects of mutations and phototoxic skin erythema in contrast to PUVA. ${ }^{69}$ VigiAccess ${ }^{56}$ reported 7 adverse effects of khellein in general and 2 of these were related to skin and subcutaneous reactions, i.e., pruritis and 4 cases related to hepatobiliary disorders. The hepatotoxicity remains the potential concern with the use of khellin. In the current systematic review Psoralens have been observed as good choice alone or in combination with phototherapy in the management of vitiligo with 
common adverse effects of pruritis, phototoxic reactions, erythema (mild-severe), itching, scaling, giddiness, nausea, depigmented macules, hyperpigmentation and gastrointestinal disturbances. Concerning the reports of adverse events of psoralens, VigiAccess ${ }^{56}$ reported 34 cases of total adverse events including 6 cases of skin and subcutaneous reactions (macule, psoriases, skin disorder, skin exfoliation, skin hyperpigmentation, skin lesion, skin toxicity and urticaria). Over the centuries it has been believed that medicinal plants containing psoralens when combined with phototherapy can successfully benefit vitiligo patients ${ }^{70}$ However most of the observers came across the negative results of blisters, pruritis, itching, urticaria and erythema, etc., by adopting this therapeutic strategy. ${ }^{71}$ Lentigines (little brown patches on skin) a side effect has been associated with extensive use of PUVA therapies in old males of skin type V and VI. Once appeared, these PUVA induced lentigines do not subside after the discontinuation of PUVA therapy although it has been believed to be reversed with cryotherapy (cold therapy with an instrument called cryoprobe using liquid nitrogen or nitrous oxide where abnormal tissues are frozen and destroyed with the therapy). ${ }^{72}$. Dilute solution of PUVA in water is termed as bath PUVA having promising advantages over topicals and oral uses of PUVA. Its delivery to the skin is uniform thus avoidance of localized phototoxic reactions as well as uneven pigmentation. ${ }^{73}$ Bath PUVA reduces the long term risk of squamous cell carcinoma by reducing cumulative doses of UVA, trade off is the incidence of more common phototoxic reactions. ${ }^{74}$ There are some reports of inflammatory hyperpigmentation induced by Demodex mites (Demodex folliculorum and Demodex brevis) hence the researcher may also keep in mind the such hyperpigmentation when monitoring the clinical studies related to herbs. ${ }^{75}$ Friction has been mentioned as a distinct aetiology of hyperpigmentation in Indian patients $^{76}$ and Demodex inflammation has been associated to induce the frictional melanosis in influencing the hyperpigmentation. ${ }^{77}$ Herbal Taraxaci, Herba Agrimoniae and Cortex Phellodendri et al have remarkable anti-mites potential along with human skin safety versus the Demodex folliculorum. ${ }^{78,79}$ Based on in vivo melanocyte proliferation potential of Piper nigrum extract (containing piperine) ${ }^{80}$ there has been a clinical human study on few patients using the piperine extract, piperine alone or piperine extract associated with prostaglandins. Pigmentation effects were reported with such therapies and latter combination speeded up the pigmentation with changed pigmentation pattern. ${ }^{81}$ The results provide a clue of future benefits of such uses if the controlled clinical studies are carried out on larger batches of patients as the existing data involve few patients ( 3 patients only) and 1 patient of the 3 had withdrawn from the study due to intense burning sensations, irritation and local redness. The 2 remaining patients reported a slight burning sensation on first use under an occlusive dressing. The clinical study was not controlled and the application of ointment with rubbing can give false results due to frictional melanosis. Furthermore the formulation development and characterization of the ointment before loading the drug has not been mentioned in this study on two patients.

\section{Limitations of the Literature Search}

It is possible that not all plants were identified (in Phase 1) and that not all relevant papers were found in the second phase literature search.

\section{Conclusion}

Primarily the retrieved clinical studies were efficacy oriented and safety parameters were secondary in priority whilst the general protocol of clinical trials requires the screening of drugs/medicinal plants on the basis of safety studies before testing the clinical aspect of efficacy. Thereby it is recommended that efficacy studies may be followed once the safety has been established for a particular medicinal plant in treating vitiligo and hypermelanosis.

\section{Recommendations}

The author recommends similar studies related to safety of medicinal plants and reports of harm for skin ailments other than vitiligo and hypermelanosis.

\section{Acknowledgment}

Work was undertaken while author was a visiting scholar in the School of Pharmacy, University of Auckland, New Zealand from May 2017 to October 2017. The author is thankful to Professor Dr Zaheer-Ud-Din Babar for his reference and support in getting the guidance of relevant herbal medicine expert at the University of Auckland. Databases use expert Sue Fooggin is regarded for her help in the literature search. Higher Education Commission (HEC) Pakistan is acknowledged for supporting the research. . 


\section{Disclosure}

The author reports no conflicts of interest for this work. A part of this article was made available as pre-print on research gate and will be updated accordingly once published in the journal.

\section{References}

1. Khandalavala BN, Nirmalraj MC. Rapid partial repigmentation of vitiligo in a young female adult with a gluten-free diet. Clin Dermatol. 2014;6(3):283-287.

2. Torok HM. A comprehensive review of the long-term and short-term treatment of melasma with a triple combination cream. Am J Clin Dermatol. 2006;7:223-230. doi:10.2165/00128071-20060704000003

3. Khushboo PS, Jadhav VM, Kadam VJ, et al. Psoralea corylifolia linn. - "kushtanashini". Pharmacogn Rev. 2010;4(7):69-76. doi:10.4103/0973-7847.65331

4. Szczurko O, Born HS. A systematic review of natural health product treatment for vitiligo. BMC Dermatol. 2008;8:2. doi:10.1186/14715945-8-2

5. Fisk WA, Agbai O, Lev-Tov HA, Sivamani RK. The use of botanically derived agents for hyperpigmentation: a systematic review. $J$ Am Acad Dermatol. 2014;70(2):352-365. doi:10.1016/j. jaad.2013.09.048

6. Ekor M. The growing use of herbal medicines: issues relating to adverse reactions and challenges in monitoring safety. Front Pharmacol. 2013;4:177.

7. Masoumeh G, Ali E. Clinical efficacy of liposome-encapsulated Aloe vera on melasma treatment during pregnancy. J Cosmet Laser Ther. 2017;19(3):181-187. doi:10.1080/14764172.2017.1279329

8. Shahla K, Ali A, Hossein M, et al. The efficacy of topical use of petroselinum crispum (parsley) versus hydroquinone cream for reduction of epidermal melasma: a randomized clinical trial. AJP. 2015;5(1):124-125.

9. Debbie M, Palmer DO, Jennifer SK. A double blind, randomized, controlled clinical trial evaluating the efficacy and tolerance of a novel phenolic antioxidant skin care system containing Coffea arabica and concentrated fruit and vegetable extracts. J Drugs Dermatol. 2010;9(12):1480-1487.

10. Buggiani G, Tsampau D, Hercogovà J, et al. Clinical efficacy of a novel topical formulation for vitiligo: compared evaluation of different treatment modalities in 149 patients. Dermatol Ther. 2012;25(5):472-476. doi:10.1111/j.1529-8019.2012.01484.x

11. Hussain I, Hussain I, Manan A, et al. Fabrication of anti-vitiligo ointment containing Psoralea corylifolia: in vitro and in vivo characterization. Drug Des Devel Ther. 2016;10:3805-3816. doi:10.2147/DDDT.S114328

12. Orest S, Neill S, Anna T, et al. Ginkgo Biloba for the treatment of vitiligo Vulgaris: an open label pilot clinical trial. BMC Complement Altern Med. 2011;21:1-9.

13. Ahmed R, Abu R, Noor MA, et al. Evaluation of standardized extract of Ginkgo Biloba in vitiligo remedy. Asian J Pharm Clin Res. 2013;6 (5):127-130.

14. Parasad P. Effectiveness of oral Ginkgo biloba in treating limited, slowly spreading vitiligo. Clin Exp Dermatol. 2003;28:285-287. doi:10.1046/j.1365-2230.2003.01207.x

15. Jun Y, Atsushi S, Shoichi T, et al. Oral intake of proanthocyanidin-rich extract from grape seeds improves chloasma. Phytother Res. 2004;18:895-899. doi:10.1002/ptr.1537

16. Beidi KL, Usha Z, Chopra CL. Picrorhiza kurroa, an ayurvedic herb, may potentiate photochemotherapy in vitiligo. J Ethnopharmacol. 1989;27:347-352. doi:10.1016/0378-8741(89)90009-3
17. Glen A, Nino C, Ailynne V, et al. Maria Jasmin Jamora MD. A comparative study of the safety and efficacy of $75 \%$ Mulberry (Morus alba) Extract oil versus placebo as a topical treatment for melasma: a randomized, single-blind, placebo-controlled Trial. J Drugs Dermatol. 2011;10(9):1025-1031.

18. Ammar M, Ahmed M, Isha L, et al. randomized, double-blinded, placebo controlled trial of oral Polypodium leucotomos extract as an adjunct to sunscreen in the treatment of melasma. JAMA Dermatol. 2013;149(8):981-983. doi:10.1001/jamadermatol.2013.4294

19. Lucy K, Martin M. A randomized double-blind placebo controlled study evaluating the effectiveness and tolerability of oral polypodium leucotomos in patients wit melasma. J Am Acad Dermatol. 2012; Ab21:4630.

20. Eduardo R, Pedro J, Elena H, et al. Systemic Immunomodulatory effects of Polypodium leucotomos as an adjuvant to PUVA therapy in generalized vitiligo: a pilot study. J Dermatol Sci. 2006;41:213-216. doi:10.1016/j.jdermsci.2005.12.006

21. Martiza A, Middelkamp H, Madhu A, et al. Orally administered Polypodium leucotomos extract decreases psoralen UVA induced phototoxicity, pigmentation, and damage of human skin. $J$ Am Acad Dermatol. 2004;50:41-49. doi:10.1016/S0190-9622(03)02732-4

22. Mark S, Nestor BD, Nicole S. Safety and efficacy of oral Polypodium leucotomos extract in healthy adult subjects. J Clin Aesthet Dermatol. 2015;8(2):19-23.

23. Colucci R, Dragoni F, Conti R. Evaluation of an oral supplement containing Phyllanthus emblica fruit extracts, vitamin $E$, and carotenoids in vitiligo treatment. Dermatolog Treat. 2015;28:17-21. doi: $10.1111 /$ dth. 12172

24. Adilson C, Tatiana C, Juliana M. Association of emblica, licorice and belides as an alternative to hydroquinone in the clinical treatment of melasma. An Bras Dermatol. 2010;85(5):613-620. doi:10.1590/ S0365-05962010000500003

25. Ni Z, Mu Y, Gulati O. Treatment of melasma with pycnogenol. Phytother Res. 2002;16:567-571. doi:10.1002/ptr.1085

26. Mendoza CG. A randomized, double blind, placebo-controlled clinical trial on the efficacy of 3\% Rumex occidentalis cream versus $4 \%$ hydroquinone cream in the treatment of melasma among Filipinos. Int J Dermatol. 2014;53:1412-1416. doi:10.1111/ijd.12690

27. Morag M, Nawrot J, Siatkowski I, et al. A double-blind, placebocontrolled randomized trial of Serratulae quinquefoliae folium, a new source of $\beta$-arbutin, in selected skin hyperpigmentations. $J$ Cosmet Dermatol. 2015;14(3):185-190. doi:10.1111/jocd.12147

28. Leeuw JD, Assen YJ, Van N, et al. Treatment of vitiligo with khellin liposomes, ultraviolet light and blister roof transplantation. $J$ Eur Acad Dermatol Venereol. 2011;25(1):74-81. doi:10.1111/j.14683083.2010.03701.x

29. Saraceno R, Steven P. Nistico, monochromatic excimer light $308 \mathrm{~nm}$ in monotherapy and combined with topical khellin $4 \%$ in the treatment of vitiligo: a controlled study. Dermatolog Treat. 2009;25:391-394. doi:10.1111/j.1529-8019.2009.01252.x

30. Valkova TC. Treatment of vitiligo with local Khellin and UVA: comparison with systemic PUVA. Clin Exp Dermatol. 2003;29:180-184. doi:10.1111/j.1365-2230.2004.01462.x

31. Orecchia G, Sangalli M, Gazzaniga A, Giordano F. Topical photochemotherapy of vitiligo with a new Khellin formulation. J Dermatolog Treat. 1998;9(2):65-69. doi:10.3109/09546639809161375

32. Procaccini E, Riccio G, Monfrecola G. Ineffectiveness of topical Khellin in photochemotherapy of vitiligo. $J$ Dermatolog Treat. 1995;6(2):117-120. doi:10.3109/09546639509097164

33. Bernhard O, Adrian T, Herbert H. Treatment of vitiligo with Khellin and Ultraviolet A. J Am Acad Dermatol. 1988;18:693-701. doi:10.1016/S0190-9622(88)70092-4

34. Mofty M, Bosseila M, Mashaly HM, et al. Broadband ultraviolet A vs. psoralen ultraviolet $A$ in the treatment of vitiligo: a randomized controlled trial. Clin Exp Dermatol. 2013;38 (8):830-835. doi:10.1111/ced.12099 
35. Shivani B, Bijaylaxmi S, Vijay G. Psoralen-narrowband UVB phototherapy for the treatment of vitiligo in comparison to narrowband UVB alone. Photodermatol Photoimmunol Photomed. 2013;29:311-317. doi:10.1111/phpp. 12072

36. Ranjeeta S, Sudha A, Tapan K. Systemic PUVA vs narrowband UVB in the treatment of Vitiligo: a randomized controlled Study. Int $J$ Dermatol. 2012;51:1107-1115. doi:10.1111/j.13654632.2011.05454.x

37. Yones SS, Palmer RA, Garibaldinos TM, Hawk JL. Randomized double-blind trial of treatment of Vitiligo: efficacy of psoralen-UVA therapy vs narrowband-UV-B therapy. Arch Dermatol. 2007;143:578-584. doi:10.1001/archderm.143.5.578

38. Bhatnagar A, Kanwar AJ, Parsad D, De D. Psoralen and ultraviolet $\mathrm{A}$ and narrow-band ultraviolet $\mathrm{B}$ in inducing stability in vitiligo, assessed by vitiligo disease activity score: an open prospective comparative study. $J$ Eur Acad Dermatol Venereol. 2007;21 (10):1381-1385. doi:10.1111/j.1468-3083.2007.02283.x

39. Ermis A. Is the efficacy of Psoralen plus ultraviolet A therapy for vitiligo enhanced by concurrent topical calcipotriol? A placebo-controlled double-blind study. $\mathrm{Br} J$ Dermatol. 2001;145:472-475. doi:10.1046/j.1365-2133.2001.04286.x

40. Ameen E, Chu A. Topical calcipotriol as monotherapy and in combination with psoralen plus ultraviolet $\mathrm{A}$ in the treatment of vitiligo. $\mathrm{Br}$ $J \quad$ Dermatol. 2001;145:476-479. doi:10.1046/j.13652133.2001.04381.x

41. Parsad D, Saini R, Verma N. Combination of PUVAsol and topical calcipotriol in Vitiligo. Dermatology. 1998;197(2):167-170. doi: $10.1159 / 000017991$

42. Parasad D, Roma P, Sunil D, et al. Clinical study of repigmentation patterns with different treatment modalities and their correlation with speed and stability of repigmentation in 352 vitiliginous patches. $J$ Am Acad Dermatol. 2004;50(1):63-67. doi:10.1016/S01909622(03)00786-2

43. Wiete W, Ludmila N. Treatment of vitiligo with UV-B radiation vs topical psoralen plus UV-A. Arch Dermatol. 1997;133:1525-1528. doi:10.1001/archderm.1997.03890480045006

44. Mofty A, Hussein E. Clinical study of a new preparation of 8-methoxypsoralen in photochemotherapy. Int J Dermatol. 1994;33 (8):588-593. doi:10.1111/j.1365-4362.1994.tb02904.x

45. Africk J, Fulton J. Treatment of vitiligo with topical trimethylpsoralen and sunlight. Br J Dermatol. 1989;84:151-156. doi:10.1111/ j.1365-2133.1971.tb06858.x

46. Sehgal. A comparative clinical evaluation of trimethylpsoralen, psoralen and 8-methoxypsoralen in treating vitiligo. Int $J$ Dermatol. 1975;14:205-208. doi:10.1111/ijd.1975.14.3.205

47. Mofty AME, Nada MM. On the treatment of vitiligo. Int J Dermatol. 1971;10(4):262-266. doi:10.1111/j.1365-4362.1971.tb01709.x

48. Dushet P, Schwarz T, Pusch M, Gshnait F. Marked increase of liver transaminases after khellin and UVA therapy. $J$ Am Acad Dermatol. 1989;21(3):592-593. doi:10.1016/S0190-9622(89)80248-8

49. Kreuter A, Gambichier AA, Jansen T. Localized vitiligo successfully treated with cream-psoralen + Ultraviolet A. J Eur Acad Dermatol Venereol. 2001;15:357-358.

50. Maurice CJ. The dangers of herbalism. BMJ. 1989;299:1204. doi:10.1136/bmj.299.6709.1204

51. David W, Mai CO. Childhood vitiligo successfully treated with Bath PUVA. Pediatr Dermatol. 1998;15(1):53-55. doi:10.1046/j.15251470.1998.1998015053.x

52. Zouhir D. Successful treatment of facial vitiligo with honey bee, Allium cepa and Avena sativa combined to sun light exposure: a case clinical trial. Int J Pharm Clin Res. 2015;7(1):9-14.

53. Rigmor M, Jette H. Methoxsalen and multiple basal cell carcinomas. Arch Dermatol. 1976;112:1613-1614. doi:10.1001/ archderm.1976.01630350079027
54. Deborah AS, Stwart M. A rare case of acute hepatitis induced bu use of Babchi seeds as an ayurvedic remedy for vitiligo. BMJ Case Rep. 2014;1-3. doi:10.1136/bcr-2013-200958

55. Abdel N, Okby WM. Psoralen plus ultraviolet A irradiation-induced lentigines arising in vitiligo: involvement of vitiliginous and normal appearing skin. Clinical Dermatol. 2004;29:380-382.

56. World Health Organization.TM.VigiAccessAvailable from: www.vig iaccess.org. Accessed December 9, 2017.

57. Donata SR, Kesavan M, Austin KS, Rajagopalan K, Kuttan R. Clinical trial of certain ayurvedic medicines indicated in vitiligo. Anc Sci Life. 1990;9:202-206.

58. Psoralea corylifolia. Available from: http://examine.com/supple ments/Psoralea+Corylifolia/summary2-1. Accessed 22 Jul 2013.

59. New WB. Manual of Homeopathic Materia Medica and Repertory. 9th ed. New Delhi: B. Jain Publishers Pvt. Ltd; 2002:11-29.

60. Stephen B, Harley G, Amy P, et al. Spontaneous bleeding associated with Ginkgo biloba. A case report and systematic review of the literature. J Gen Intern Med. 2005;20:657-661. doi:10.1007/s11606005-0114-4

61. Ansari R, Aswal B, Chander R, et al. Hepatoprotective activity of kutin- the iridoid glycoside mixture of Picrorhiza Kurroa. Indian J Med Res. 1988;87:401-404.

62. Handa S, Sharma A, Chakraborty K. Natural products and plants as liver protecting drugs. Fitoterapia. 1986;58:307-351.

63. Middelkamp-Hup MA, Pathak MA, Parrado C, et al. Oral Polypodium leucotomos extract decreases ultraviolet-induced damage of human skin. J Am Acad Dermatol. 2004;51:910-918. doi:10.1016/j.jaad.2004.06.027

64. Gonzales S, Pathak MA, Cuevas J, Villarrubia VG, Fitzpatrick TB. Topical or oral administration with an extract of Polypodium leucotomos prevents acute sunburn and-psoralen induced phototoxic reactions as well as depletion of Langerhans cells in human skin. Photodermatol Photoimmunol Photomed. 1997;13:50-60. doi:10.1111/j.1600-0781.1997.tb00108.x

65. Anrep GV, Kenawy MR, Barsoum GS. The coronary vasodilator action of khellin. Am Heart J. 1949;37:531-542. doi:10.1016/00028703(49)91131-X

66. Herbert Hbnigsmann MD, Bernhard Ortel MD, Tanew A. Reply. J Am Acad Dermatol. 1989;21(3):593-594. doi:10.1016/S01909622(89)80249-X

67. Armbrust CA, Levine SA. The treatment of angina pectoris with a preparation of khellin. Am J Med Sci. 1950;220:127. doi:10.1097/ 00000441-195008000-00002

68. Hofer A, Karl H, Wolf P. Long-term results in the treatment of vitiligo with oral khellin plus UVA. Eur $J$ Dermatol. 2001;11:225-229.

69. Morliere P, Honigsmann H, Averbeck D. Phototherapeutic, photobiologic and photosensitizing properties of Khellin. J Invest Dermatol. 1988;90:720-724. doi:10.1111/1523-1747.ep13083852

70. Ortonne J. Psoralen therapy in vitiligo. Clin Dermatol. 1989;7 (2):120-135. doi:10.1016/0738-081X(89)90062-X

71. Said HM. Hamdard pharmacographica. Indica. 1972;XV(Special Issue):412-413.

72. Leroy D, Dompmartin A, Dubreuil A, et al. Cryotherapy of PUVA lentigines. Br J Dermatol. 1996;135:988-990. doi:10.1046/j.13652133.1996.d01-1108.x

73. Collins P, Rogers S. Bath water compared with oral delivery of 8-methoxypsoralen PUVA for chronic plaque type psoriasis. $\mathrm{Br}$ $J$ Dermatol. 1992;127:392-395. doi:10.1111/j.1365-2133.1992. tb00460.x

74. Ortel B, Perl S, Kinaciyan T, et al. Comparison of narrow-band (311 nm). UVB and broad band UVA after oral or bath water 8-methoxypsoralen in the treatment of Psoriasis. J Am Acad Dermatol. 1993;29:736-740. doi:10.1016/0190-9622(93)70239-P 
75. Forton F, Germaux MA, Brasseur T, et al. Demodicosis and rosacea: epidemiology and significance in daily dermatologic practice. $\mathrm{J} \mathrm{Am}$ Acad Dermatol. 2005;52:74-87. doi:10.1016/j.jaad.2004.05.034

76. mutalik SD, Pethe SV, Nikam BP, et al. Facial frictional melanosis in Indian patients: defining the entity. Clin Dermatol Rev. 2019;3 (1):78-83. doi:10.4103/CDR.CDR_6_18

77. Shekhaar N, Radhakrishnan PK. Demodex induced facial frictional melanoses. Aust J Dermatol. 2020;61(4):e490-e492.

78. Tian Y, Li CP, Deng Y. Anti-mite activity and skin safety of Herbal taraxaci extract for Demodex folliculorum. Zhongguo Ji Sheng Chong Xue Yu Ji Sheng Chong Bing Za Zhi. 2007;25(2):133-136.
79. Tian Y, Li CP. Anti-mite activities of 25 kinds of traditional Chinese medicines for Demodex folliculorum]. Zhong Yao Cai. 2006;10:1013-1015.

80. Faas L, Venkatasamy R, Hider RC, Young AR, Soumyanath A. In vivo evaluation of piperine and synthetic analogues as potential treatments for vitiligo using a sparsely pigmented mouse model. $\mathrm{Br}$ J Dermatol. 2008;158:941-950. doi:10.1111/j.13652133.2008.08464.x

81. Mihăilă B, Dinică RM, Tatu A, et al. New insights in vitiligo treatments using bioactive compounds from Piper nigrum. Exp Ther Med. 2019;17(2):1039-1044. doi:10.3892/etm.2018.6977

\section{Publish your work in this journal}

Clinical, Cosmetic and Investigational Dermatology is an international, peer-reviewed, open access, online journal that focuses on the latest clinical and experimental research in all aspects of skin disease and cosmetic interventions. This journal is indexed on CAS.
The manuscript management system is completely online and includes a very quick and fair peer-review system, which is all easy to use. Visit http://www.dovepress.com/testimonials.php to read real quotes from published authors. 